\title{
Autophagy in DG engrams mediates Rac1-dependent forgetting during aging via microglia-mediated synapse elimination
}

Zhilin Wang ${ }^{1,2}$, Ruyan Chen ${ }^{1,2}$, Qing Lin ${ }^{1}$, Yan Jiang, Lan $\mathrm{Ma}^{1, *}$ and Feifei Wang ${ }^{1, *}$

${ }^{1}$ Department of Neurosurgery, Huashan Hospital, State Key Laboratory of Medical Neurobiology and MOE Frontiers Center for Brain Science, School of Basic Medical Sciences, Institutes of Brain Science, Fudan University, Shanghai, 200032, China

${ }^{2}$ These authors contributed equally to this work.

*Corresponding author. Email: lanma@fudan.edu.cn and ffwang@fudan.edu.cn. 


\section{Abstract}

Engrams are considered to be substrates for memory storage, and the functional dysregulation of the engrams leads to cognition impairment. However, the pathological changes of the engrams leading to forgetting, which typically involves a failure in memory retrieval, remains unclear. Here we found that the expression of autophagy protein 7 (Atg7) in dentate gyrus (DG) engrams was dramatically increased in aged mice, leading to the activation of surrounding microglia and impair retrieval of conditioned fear memory. Using transcriptomic and fluorescence in situ hybridization analyses, we demonstrated Toll-like receptor (TLR) pathway were upregulated in DG microglia by overexpressing ATG7 in DG engrams. TLR2/4 in the microglia mediates the excessive synapse elimination and impaired retrieval of fear memory induced by ATG7-depedent autophagy in DG engrams. The expression of Rac1, a Rho-GTPases which mediates active forgetting, was upregulated in aged engrams. Optogenetic activation of Rac1 in DG engrams promoted the expression of ATG7 and autophagy in the engrams, the activation of microglia, and thus impaired the retrieval of fear memory. Interference of the $\operatorname{Atg} 7$ expression in the engram and microglia activation prevented the impairment of fear memory retrieval induced by activation of Rac1 in DG engrams. Together, our findings revealed autophagy-dependent remodeling of DG engrams by microglia as a novel interference mechanism of memory retrieval. 


\section{Introduction}

Memory, the ability to store learned information, is an essential property of the brain. A given memory is encoded by sparsely distributed engrams which are a population of neurons that become highly activated during learning (Holtmaat and Caroni, 2016). The regulation of engrams in the brain has been viewed as an exclusive prerogative of the neuronal system. In addition to neurons, however, the brain comprises other types of cells, including glia and vasculature. Recent findings support these microglia, the major immune cells in the brain, are closely associated with the neural remodeling from rodents to human in an experience-dependent manner (Cangalaya et al., 2020; Cheadle et al., 2020a; Cong et al., 2020; Wu et al., 2015). Microglia were involved in both neurogenesis-related and aging-dependent memory destabilization (Barrientos et al., 2010; Spittau, 2017; Wang et al., 2020a). C1q-dependent complement pathway is actively involved in synapse elimination by microglia in adult hippocampus (Wang et al., 2020a). High resolution imaging shows that microglia engulf retinal ganglion cell inputs during the period of activity-dependent synaptic pruning during the early stage of postnatal development (Schafer et al., 2012). The cellular mechanisms that underlie synaptic remodeling mediated by microglia is crucial for figuring out the possible pathogenesis of psychiatric disorders due to the impaired synaptic refinement, such as Alzheimer disease (Hammond et al., 2019; Rajendran and Paolicelli, 2018), schizophrenia (Osimo et al., 2019; Sellgren et al., 2019) and autism (Heavner and Smith, 2020).

Previous researches have shown that microglia-neuron interaction is necessary for neuronal remodeling and memory precision. Microglia become morphologically and functionally activated by some trigger molecules that believed to be derived from the neurons. Growing evidence demonstrate that the binding between $\mathrm{CX} 3 \mathrm{C}$ chemokine receptor 1 (CX3CR1) expressed on microglia and 
CX3CL1 secreted from neurons attributes to the neuron-microglia communication. Impaired CX3CL1/ CX3CR1 signaling is involved in the pathogenesis of Alzheimer's disease (Fuhrmann et al., 2010). Recently, it is demonstrated that Toll-like receptors (TLRs) are activated by pathogen-associated molecular patterns (PAMPS), and play an important role in host defense response in the CNS (Fiebich et al., 2018; Kawasaki and Kawai, 2014). The activation of TLRs in microglia have been observed in both Alzheimer's and Parkinson's patients (Fiebich et al., 2018). TLRs-mediated signaling pathways in microglia lead to the translocation of transcription factors, such as NF- $\kappa \mathrm{B}$, thereby activating the transcription of several genes including interleukins (ILs) and tumor necrosis factors (TNFs) which mediated synaptic pruning and functional plasticity in neurons (Lewitus et al., 2016; Liao et al., 2016; Werneburg et al., 2017).

Growing evidence shows that cell debris or signaling molecules are transferred from neurons to glia to survey neuronal state (Kulkarni et al., 2018; Stowell et al., 2019). Autophagy is a complex self-degradative system that removes damaged cellular debris and aggregated proteins to maintain brain homeostasis and promote neuronal survival (Glick et al., 2010). Autophagy maintains neuronal homeostasis through degrading and recycling the damaged cellular components (Kulkarni et al., 2018). Numerous researches have shown that autophagy is involved in microglia activation in an unconventional secretion pathway (Kulkarni et al., 2018). Recent reports found that autophagy mediated unconventional secretion of cytosolic organelles and a series of cytosolic proteins (Gonzalez et al., 2020). Previous researches shown autophagy is upregulated in the hippocampus when mice were exposed to contextual fear conditioning (CFC), Morris water maze tasks and novel object recognition (Glatigny et al., 2019). However, whether autophagy in engrams affects memory retrieval through activating microglia in an unconventional secretion way remains unclear. 
In this study, we found that autophagy protein 7 (Atg7) mRNA in the dorsal dentate gyrus (DG) engrams which encodes fear memory, was increased in aged mice, leading to the impairment of fear memory retrieval through TLR2/4-depedent activation of microglia. In addition, Rac1-mediated forgetting signal promoted autophagy in DG engrams. These signaling crosstalk between engrams and microglia lead to synapse elimination of engrams and memory destabilization, which might be a potential interference mechanism of memory retrieval in aging mice.

\section{Results}

The expression of ATG7 is increased in dorsal DG engrams in aged-mice, impairs the retrieval of contextual fear memory, and activates DG microglia.

Impaired memory maintenance and increased autophagy of the neuron has been found in Alzheimer's disease (AD) and aging animal model (Bordi et al., 2016). To assess the autophagy in dorsal DG engram, we used a doxycycline (Dox)-dependent robust activity marking (RAM) system to label the engrams(Sorensen et al., 2016). Aged (24-month-old) mice and young (3-month-old) mice were infected with $A A V-R A M-E G F P$ in dorsal dentate gyrus (DG) to label the engrams during fear conditioning (Fig 1A). 3 days later after engrams labeling, mice were exposure to context A (the fear conditioned context), and c-Fos immunostaining was performed to assess the reactivation of $\mathrm{EGFP}^{+}$engrams. The total number of $\mathrm{EGFP}^{+}$engrams in $\mathrm{DG}$ and the proportion of c-Fos ${ }^{+}$engrams $\left({\mathrm{c}-\mathrm{Fos}^{+}}^{+} \mathrm{EGFP}^{+}\right)$were decreased in aged mice, compared with that in the young mice (Fig 1B), indicating the reactivation of the engrams during the memory retrieval were decreased in aged mice. We then assess the level of autophagy in DG engrams by performing single molecule fluorescence in situ hybridization (sm FISH) against $\operatorname{Atg} 7$ (Autophagy Related Protein 7) and Egfp mRNA. The $\operatorname{Atg} 7$ 
mRNA abundance in $E g f p^{+}$engrams were significantly increased in aged mice, compared with the young mice (Fig 1C-D). Autophagy could be specifically manipulated by overexpression or downregulation of ATG7 (Hui et al., 2019; Kim et al., 2017). To assess the role of autophagy in DG engram in memory retrieval, mice were infected with AAV-RAM-Cre, AAV-DIO-Atg7-shRNA-EGFP (or DIO-Scramble-shRNA) in dorsal DG to specifically knock down Atg7 in the engrams (Fig 1E). The expression of $A \operatorname{tg} 7 \mathrm{mRNA}$ was dramatic decreased in $\operatorname{Atg} 7-\operatorname{sh} R N A$ expressing $\left(\mathrm{EGFP}^{+}\right)$engrams (Fig 1F). Knocking down ATG7 in DG engrams increased the freezing level in the conditioning context, indicating the enhanced retrieval of contextual fear memory (Fig 1G-H). Instead, mice were infected with AAV-RAM-Cre, AAV-DIO-Atg7-Flag (or DIO-EYFP) in dorsal DG to specifically overexpress ATG7 in the engrams. Immunostaining of DG showed a dramatic increase of phagocytic marker CD68 puncta within $\mathrm{Iba}-1^{+}$microglia in mice overexpressing ATG7, compared with the EYFP group, indicating that ATG7 in DG engrams might increase the activation of the microglia (Fig 1K). Colony-stimulating factor 1 receptor (CSF1R) signaling is crucial for microglial survival (Spangenberg et al., 2016). Mice were treated with diet containing Pexidartinib (PLX3397) (CSF1R inhibitor) (Wang et al., 2020a) to deplete microglia. Significantly elimination of the microglia in the DG were observed 3 days after PLX3397 administration (Fig 1L-M). Overexpression of ATG7 in DG engrams impaired the retrieval of contexture fear memory in mice, while PLX3397 pretreatment prevented ATG7-induced impairment of memory retrieval (Fig 1N-O). These results demonstrate that ATG7 in DG engrams activates microglia and impairs the retrieval of contexture fear memory.

\section{TLR2/4 in microglia mediates ATG7-induced spine elimination in DG engrams}

To determine mechanism of microglia activation by autophagy in DG engrams, the mice were 
infected with $A A V$-RAM-Cre, $A A V$-DIO-Atg7-Flag or $A A V$-DIO-EYFP in dorsal DG to overexpress ATG7 in the DG engrams. The dorsal DG was dissected 3 days after engrams labeling, and microglia were enriched using magnetic-activated cell sorting (MACS) (Fig 2A). Fluorescence-activated cell sorting analysis were performed to assess the purity of the enriched microglia (Fig 2B). RNA-Seq and Gene Ontology (GO) of biological process categories with enrichment showed that when overexpression of ATG7 within DG engrams, the upregulated genes in DG microglia were enriched in immune response pathways, including $\mathrm{T}$ cell activation and cytokine-mediated signaling pathway (Fig 2C-D). Gene Set Enrichment Analysis (GSEA) revealed that TLR signaling pathway was upregulated in microglia when engrams overexpressed ATG7, indicating the positive correlation between ATG7 overexpression within engrams and TLR signaling upregulation within microglia (Fig 2E). When analyzed the genes involved in immune response, the expression of Toll-like receptor 2 (Tlr2), the most abundantly TLR family member expressed in microglia is increased (Fig 2F). It has been well-documented that TLR2 contributed to the activation of microglia (Liao et al., 2016; Nie et al., 2018; Stirling et al., 2014). The activation of microglia through Toll-like receptor 2/4 (TLR2/4) initiates inflammatory signaling, contributes to the release of Interleukin-1 alpha (IL-1 $\alpha$ ) and tumor necrosis factor alpha (TNF $\alpha)$, which mediates synaptic pruning (Nie et al., 2018). Consistently, GSEA showed the enrichment of genes involved TNF signal pathway and cytokine-cytokine receptor interaction in microglia when engrams overexpressed ATG7 (Fig 2G), suggesting the ATG7-dependent autophagy in the engrams promote immune response in microglia. The expression Tlr2 and Tlr4 in microglia were determined by smFISH (Fig 2H). CX3C Motif Chemokine Receptor 1 (CX3CR1) were used to label the microglia. The expression of $T l r 2 / 4$ mRNA in $C x 3 c r 1^{+}$microglia of DG were upregulated by overexpression of ATG7 in the DG engrams, compared with the EYFP 
group (Fig 2I-K).

To explore whether TLR2/4 within microglia were involved in the impaired memory retrieval induced by autophagy within the engrams., we downregulated TLR2/4 in DG microglia with the lentivirus expressing Tlr2/4 microRNA or control microRNA in a Cre-dependent manner (Fenno et al., 2014b). Cx3cr1-CreER mice were infected with the $L V$-DIO-Tlr4miR-Tlr2miR-mCherry or $L V$ DIO-NegmiR-mCherry in the DG, in which Cre recombinase are selectively expressed in microglia(Nie et al., 2018) when treated with tamoxifen, and thus specifically expressed $T l r 2 / 4 m i R$ or NegmiR in microglia. Tlr2/4miR-mCherry significantly downregulate the expression of Tlr2 and Tlr4 mRNA in DG microglia isolated by MACS (Fig 3A). To determine whether upregulation of ATG7 in DG engrams interferes with the retrieval of fear memory through Tlr2/4 within microglia, Cx3cr1-CreER mice were infected with AAV-RAM-Flp, AAV-RAM-Frt-Atg7-Flag, and LV-DIO-Tlr2/4miR-mCherry (or NegmiR-mCherry) in dorsal DG (Fig 3B), in which Cre-loxp and Flp-FRT (Fenno et al., 2014a) system were combined to genetic control engrams and microglia separately. After labeling and overexpressing ATG7 in the engrams, TAM was administered to induce the expression of $T l r 2 / 4 m i R$ in the microglia of DG (Fig 3C). Overexpression of ATG7 in DG engram impaired the retrieval of contexture fear memory, whereas downregulation of TLR $2 / 4$ by lentivirus in the DG microglia impeded the impairment of fear memory retrieval (Fig 3D-E). Neuronal activity is communicated to microglia and may alter microglia-neuron interactions. To assess how TLR2/4 is involved in the interaction between engrams and microglia, $C x 3 c r 1-C r e E R$ mice were injected with AAV-RAM-Flp, AAV-RAM-Frt-Atg7-Flag, AAV-RAM-mScarlet-I-post-eGRASP, and either LV-DIO-NegmiR-EGFP or $L V$-DIO-Tlr2/4miR-EGFP in dorsal DG (Fig 3F). The dendrite of the engrams was labeled with 
mScarlet-I-post-eGRASP (Choi et al., 2018). Compared with the engrams overexpressing EYFP, the contact area between microglia and the dendrites of the engrams overexpressing ATG7 were increased, and the spine density of these engrams was decreased (Fig 3G-J). Downregulation of TLR2/4 in microglia decreased the microglia-dendrite contact area and resumed the spine density (Fig 3G-J). These data indicate that microglia contact with the engrams and promote the elimination of the spine in a TLR2/4-dependent manner.

\section{Forgetting signal Rac1 in DG engrams impairs the retrieval of fear memory and activates}

\section{autophagy within the engrams}

The activity of Ras-related C3 botulinum toxin substrate 1 (Rac1), which regulates active forgetting in both fly and mice, is aberrantly elevated in the hippocampal tissues of AD patients and AD animal models (Wu et al., 2019). An age-dependent Racl activity-based memory loss was observed in an AD fly model (Wu et al., 2019). Sm FISH showed a significant increased mRNA level of Racl within the DG engram of the aged (24-month-old) mice, indicating the age-dependent upregulation of Rac1 (Fig 4A-B). To investigate whether Rac1 activity in DG engrams impairs the retrieval of contexture fear memory, WT mice were infected with $A A V$-RAM-Cre mixed with either $A A V-D I O$ -C450A-mVenus or AAV-DIO-Pa Rac1-mVenus in bilateral dorsal DG to express photoactivatable Rac1 (Pa Rac1) or light-insensitive mutant (C450A) in the engrams. 3 days after engrams labeling, the light pulse $(150 \mathrm{~ms} ; 1 \mathrm{~Hz} ; 1 \mathrm{hr})$ was delivered into dorsal DG to opto-activate Rac1 before the retrieval of contexture fear memory (Fig 4C-D). c-Fos immunostaining was performed $1.5 \mathrm{hrs}$ after the memory retrieval. The percentage of the ${\mathrm{c}-\mathrm{Fos}^{+}}^{+}$component was decreased in the engrams overexpressing Pa-Rac1, compared with the C450A group, suggesting optical activation of Rac1 in 
DG engrams decreased the reactivation of engrams during memory retrieval (Fig 4D-F). Additionally, the retrieval of contexture fear memory was significantly impaired $1 \mathrm{hr}$ after optical activation of Pa-Rac1 in DG engram (Fig 4H), and this effect was lasted up to 24 hrs after optical activation of Pa-Rac1 (Fig 4I), indicating the Rac1 activation induced a long-term effect on memory retrieval. Instead, optical inhibition of Rac1 T17N within the engrams increased the freezing level in context A, indicating the enhanced retrieval of contexture fear memory (Fig 4K). Nevertheless, the enhancement was not observed 24 hrs after Rac1 inhibition (Fig 4L), indicates that the activation of Rac1 in DG engrams might be an autonomously process that contributes to the forgetting of fear memory.

Previous researches showed that Rac1 induces autophagy through activating JNK signal pathway, which enhance the expression of multiple ATG genes including $\operatorname{Atg} 5$ and $\operatorname{Atg} 7$ via a Foxo-dependent transcription pathway (Zhou et al., 2015). To assess whether Rac1 interferes with fear memory retrieval via promoting autophagy in DG engram, a Cre-dependent autophagy flux reporter system $A A V-D I O-R F P-G F P-L C 3$ were conducted (Castillo et al., 2013) based on different pH stability of EGFP and RFP fluorescent proteins (Fig 4M-N). $1 \mathrm{hr}$ after optical activation of Rac1 within the DG engrams, the autophagy signal was evaluated by analyzing the ratio of RFP and GFP fluorescent intensity. There was a substantial increase ratio of the RFP/Yellow puncta in the DG engrams (Fig 4O). These data suggest that age-dependent upregulation of Rac1 signaling in DG engram facilitates autophagy flux within the engrams and impairs fear memory retrieval.

\section{Activation of Rac1 within DG engram upregulates the expression of ATG7 and activates} microglia via TLR2/4 
Accumulating evidence suggest Rac1 mediates reversible forgetting. Activation of Rac1 within CA1 engrams affects memory maintenance (Lv et al., 2019). To assess the correlation of Rac1 dependent memory loss with autophagy within engrams, mice were infected with AAV-RAM-Cre, AAV-DIO -C450A-mVenus or AAV-DIO-Pa Rac1-mVenus in bilateral dorsal DG (Fig 5A). Optical activation of Rac1 in the DG engrams significantly increased the mRNA level of $\operatorname{Atg} 7$ in the DG engrams, compared with C450A control group (Fig 5B-C). CD68 and Iba1 immunostaining after optical stimulation were performed, and a significant increased intensity of CD68 puncta in the Iba1 ${ }^{+}$ microglia was observed in Pa Rac1 group (Fig 5D-E). Furthermore, smFISH following optical stimulation of Rac1 within DG engrams showed a significant increased Tlr2/4 mRNA level within microglia around the fiber tip in $\mathrm{Pa} \mathrm{Racl}$, compare with the C450A group (Fig 5F-H). To assess the causal link of Tlr2/4 in microglia in Rac1-induced microglia activation, Cx3crl-CreER mice were infected with AAV-RAM-Flp, AAV-Frt-Pa Rac1 (or Frt-C450A), and LV-DIO-Tlr2/4miR-mCherry (or-DIO-NegmiR-mCherry) in bilateral dorsal DG. After engrams labeling, the mice were injected with TAM to downregulate TLR2/4 in microglia in a Cre-dependent manner. The light pulse was delivered 3 days after labeling, and the immunohistochemistry was performed $1 \mathrm{hr}$ after optical stimulation (Fig 5I). The colocalization of Ibal with mCherry indicated the specific expression of Tlr2/4miR-mCherry in DG microglia (Fig 5J). Optical activation of Rac1 in the DG engrams significantly increased the density of CD68 puncta in the microglia surrounding the tips of the optical fibers, while knocking down TLR2/4 within microglia abolished the upregulation of CD68 puncta (Fig 5K-L). These data indicate TLR2/4 in microglia is required for microglia activation induced by Rac1-activation within the engrams. 
ATG7 within the engrams and TLR2/4 within the microglia mediates the impaired retrieval of fear memory induced by Rac1 activation in DG engrams.

To investigate the involvement of ATG7 within the DG engrams and DG microglia in the impaired retrieval of contexture fear memory induced by Rac1 activation, WT mice were infected with AAV-RAM -Cre, AAV-DIO-Atg7-shRNA-EGFP (or scramble shRNA), and AAV-DIO-Pa Rac1-mVenus (or C450A) bilaterally in dorsal DG. Optical activation of Pa Rac1 within DG engrams led to decreased freezing level in the fear conditioned context, indicating the impaired retrieval of contexture fear memory (Fig 6A-C), whereas downregulation of ATG7 in the DG engrams impeded the decreased freezing level in the fear conditioned context (Fig 6A-C), suggesting ATG7 within the engrams mediates the impaired the retrieval of fear memory induced by Rac1 activation. Administration of microglia inhibitor PLX3397 attenuated the decreased freezing level induced by optical activation of Rac1 within DG engrams (Fig 6D-F). No differences were detected in the open field or elevated plus maze tests, suggesting locomotor activity or anxiety level in mice were not affected by Rac1 activation or microglia depletion (Fig 6G-H). Furthermore, in Cx3crl-CreER mice, knocking down TLR2/4 within DG microglia prevented the impaired retrieval of contexture fear memory induced by the optical activation of Rac1 within the DG engrams (Fig 6I-K), without affecting locomotor activity or anxiety level in mice (Fig 6L-M). Collectively, our observations suggest that the activation of Rac1within DG engram impaired the retrieval of contexture fear memory via ATG7 within the DG engrams, as well as TLR2/4-dependent microglia activation in DG.

\section{Discussion}


In this study, we found that the expression of Rac1- and ATG7 in DG engrams were increased fin aged mice. The upregulation of Rac1 and ATG7 activates microglia, promotes spine remodeling, and thus impairs the retrieval of contexture fear memory. Besides, TLR2/4 within microglia is essential for autophagy-dependent crosstalk between engrams-microglia. Those result reveal a novel molecular mechanism underlying the memory destabilization in aged mice that contributed by the interaction between DG microglia and engrams (Fig 7).

\section{Autophagy In Neurodegenerative Diseases}

Autophagy is a natural self-degradative process that clean out damaged organelles and protein aggregates in the body to maintain cell homeostasis. The dysfunction of autophagy is believed to be involved in the pathogenesis of Alzheimer's disease (AD), which is a neurodegenerative disorder with deficiency in memory and cognitive functions. Genome-wide analysis showed the transcriptional upregulation of autophagy pathways in AD patients (Lipinski et al., 2010). Other researches demonstrated that autophagy kinase complexes such as BECN1-PIK3C3 were reduced in hippocampus of AD mouse model (Lachance et al., 2019). APP/PS1 mice lacking NF-E2 related factor 2 (Nrf2) had increased accumulation of multivesicular bodies, endosomes and lysosomes in neurons, leading to defective autophagy and increased Alzheimer's disease-like pathology (Joshi et al., 2015). For Parkinson's disease (PD), previously studies have implicated PINK1 and Parkin mutations induced defective mitophagy, thereby, accumulating damaged mitochondria and triggering Parkinson's Disease (PD) (Mancias and Kimmelman, 2016; Sarraf et al., 2013). Here, we found the autophagy in DG engrams which store the learned information is increased in aged mice (Fig 1D), and enhanced autophagy in DG engram by overexpressing ATG7 in young mice led to impaired 
retrieval of fear memory (Fig 10).

\section{Autophagy signaling in engrams mediates spine elimination induced by microglia activation}

Microglia-neuron signaling transduction is necessary for synaptic remodeling by microglia. Numerous researches have shown that microglia activation plays an important role in pruning of exuberant synaptic connections and maintaining brain homeostasis during development (Kim et al., 2017; Lui et al., 2016; Weinhard et al., 2018). Fn14 within thalamic neurons interacted with TWEAK signals within microglia to engage the number of spines on thalamic neurons (Cheadle et al., 2020b). Cytokine interleukin-33 (IL-33) can be released from DG neurons in an expression-dependent manner and bind to the IL-33R in microglia, thus promoting microglial engulfment of the extracellular matrix and leading to the increased spine density (Nguyen et al., 2020). Previous researches have shown the induction of autophagy enhanced secretion of the proinflammatory cytokine IL-1 $\beta$ via an unconventional secretion pathway depending on ATG5 (Dupont et al., 2011). IL-1 $\beta$ was found to drive microglia activation (Todd et al., 2019) and induce NFאB activation in microglia (Ferreira et al., 2010; Krasnow et al., 2017). Accumulative evidence indicates microglia mediates synapses remodeling and neural circuits reorganization in an experience- and learning-dependent manner (Miyamoto et al., 2016; Parkhurst et al., 2013), which is essential for memory precision throughout life. Aberrant microglial activation correlates with synapse loss and cognitive decline in AD mouse models in a complement-dependent process (Hong et al., 2016; Shi et al., 2015). Microglia deficit in synaptic engulfment causes weak synaptic transmission, impaired brain connectivity, which is tightly associated with autism and other neuropsychiatric disorders (Kim et al., 2017; Zhan et al., 2014). Here, we found that autophagy within the DG engrams activated the 
microglia (Fig 1K) to contact and eliminate the spine (Fig 3I-J), providing a new mechanism for the dysregulation between neurons and microglia under pathological condition.

\section{Microglial TLR2/4 mediates the crosstalk between the engrams and microglia}

Toll-like receptors (TLRs) in microglia acts as a surveil lancer recognizing the pathogen-associated molecular patterns (PAMPs) and endogenous ligands of host to respond to microenvironment changes. Previous evidence suggest $\alpha$-synuclein released from neurons when suffering stress, injury or other stimulation interacts with TLR2/4 in microglia, leading to microglia activation and engulfment of $\alpha$-synuclein in turn (Choi et al., 2020; Kim et al., 2013). TLR4 performs a critical role in A $\beta$-induced NLRP3 inflammasome and inhibition of TLR4/NLRP4 pathway alleviate extracellular A $\beta$-induced neuroinflammation (Liu et al., 2020). Ubiquitin-specific protease 8 (USP8) attenuates cognition deficiency and motor dysfunction in lipopolysaccharide(LPS)-induced mice models through inhibiting TLR4/MyD88/NF-kB pathway and increasing the expression of anti-inflammatory factor (Zhao et al., 2020). Recent studies have shown crucial roles of TLR4-dependent activation of microglia in neurodegenerative diseases, such as AD and PD (Fernandez-Arjona et al., 2019; Fiebich et al., 2018; Su et al., 2016; Yao et al., 2013). In our study, we found TLR2/4 within DG microglial is necessary for neuron-microglia cross-talk and autophagy-induced spine elimination by microglia (Fig 3G-J), and thus provides a potential therapeutic target for cognitive decline and new insights into the mechanisms of pathogenesis of neuronal degeneration. GSEA analysis indicated the TNF signal pathway and cytokine-cytokine receptor interaction in microglia, which mediates synaptic pruning(Nie et al., 2018), was upregulated in ATG7-overexpressing mice. The causal link between the autophagy dysregulation within the 
engrams and the release of IL-1 $\alpha$ and TNF $\alpha$ by microglia in pathologic conditions such as aging need to be investigate in future.

\section{ACKNOWLEDGEMENTS}

Funding: This work was supported by grants from the Natural Science Foundation of China (31930046 \& 82021002 to L.M., 31871021 to F.W.), and Shanghai Municipal Commission of Science and Technology (2018SHZDZX01 to L.M. and F.W.) and ZJLab.

\section{AUTHOR CONTRIBUTION}

FW, LM and ZW designed the experiments and analysed the data. WZ and RC carried out the stereotaxic surgery, behavioural tests, immunohistochemistry, and RNAscope ISH. RC and QL carried out the image quantitation. ZW constructed the viral vectors and drafted the manuscript. FW and LM revised the manuscript. FW and LM supervised the study.

\section{DECLARATION OF INTERESTS}

The authors declare no competing financial interests. 


\section{Materials and methods}

\section{Key Resources Table}

\begin{tabular}{|c|c|c|}
\hline REAGENT or RESOURCE & SOURCE & IDENTIFIER \\
\hline \multicolumn{3}{|l|}{ Antibodies } \\
\hline anti-c-Fos & Abcam & ab190289 \\
\hline anti-Flag & Abcam & ab205606 \\
\hline anti-CD68 & Bio-Rad & MCA1957 \\
\hline anti-mCherry & Thermo Fisher & M11217 \\
\hline anti-RFP & Rockland & $600-401-379$ \\
\hline anti-GFP & Rockland & $600-101-215$ \\
\hline anti-Iba1 & Wako Chemicals & 019-19741 \\
\hline goat anti-rabbit 488 & $\begin{array}{l}\text { Jackson } \\
\text { ImmunoResearch }\end{array}$ & $111-545-144$ \\
\hline goat anti-rabbit $\mathrm{Cy} 3$ & $\begin{array}{l}\text { Jackson } \\
\text { ImmunoResearch }\end{array}$ & $111-165-144$ \\
\hline donkey anti-rat 647 & $\begin{array}{l}\text { Jackson } \\
\text { ImmunoResearch }\end{array}$ & $712-605-150$ \\
\hline goat anti-rat $\mathrm{Cy} 3$ & $\begin{array}{l}\text { Jackson } \\
\text { ImmunoResearch }\end{array}$ & $112-165-167$ \\
\hline CD11b-PE & Thermo Fisher & $12-0112-81$ \\
\hline CD45-FITC & BioLegend & 103108 \\
\hline \multicolumn{3}{|l|}{ Chemicals, Peptides, and Recombinant Proteins } \\
\hline PLX3397 & Selleck & S7818 \\
\hline DAPI & Roche & 10236276001 \\
\hline TRIzol LS & Invitrogen & 15596026 \\
\hline Tamoxifen & Sigma & T5648 \\
\hline doxycycline & Sigma & D3447 \\
\hline \multicolumn{3}{|l|}{ Bacterial and Viral Strain } \\
\hline pAAV-Ef1a-DIO EYFP & addgene & 27056 \\
\hline pTriEx-mVenus-PA-Rac1 & addgene & 22007 \\
\hline pTriEx-mVenus-PA-Rac1-T17N & addgene & 22017 \\
\hline pTriEx-mVenus-PA-Rac1-C450A & addgene & 22021 \\
\hline ptfLC3 & addgene & 21074 \\
\hline pCAG-Cre & addgene & 13775 \\
\hline pLV-PGK-DIO-mCherry-TLR4miR-TLR2miR & addgene & 137733 \\
\hline pLV-PGK-DIO-mCherry-nega miR & addgene & 137732 \\
\hline pAAV-EF1a-Flpo & addgene & 55637 \\
\hline pAAV-Ef1a-fDIO hChR2(H134R)-EYFP & addgene & 55639 \\
\hline pAAV-RAM-EGFP & addgene & 84469 \\
\hline
\end{tabular}




\begin{tabular}{lll} 
pAAV-TIWB-myrmScarlet-I-P2A-post-eGRASP & addgene & 111584 \\
pAAV-CMV-bGlobin-Flex-EGFP-MIR30shRNA & Obio Technology & \\
\hline Critical Commercial Assays & & \\
\hline RNAscope® Multiplex Fluorescent Detection Kit v2 & ACD & Cat No.323110 \\
\hline RNAscope® 2.5 Universal Pretreatment Reagents & ACD & Cat No.322380 \\
\hline Adult Brain Dissociation Kit & Miltenyi Biotec & 130-107-677 \\
\hline CD11b microBeads & Miltenyi Biotec & 130-093-634 \\
\hline PrimeScript RT reagent Kit & Takara & RR037A \\
\hline SYBR Premix Ex Taq & Takara & RR420A \\
\hline VAHTS mRNA-seq V3 Library Kit & Vazyme & NR611-01 \\
\hline TLR4-C1 & ACD & Cat No. 316801 \\
\hline ATG7-C2 & ACD & Cat No. 561261-C2 \\
\hline EGFP-C2 & ACD & Cat No. 400281 \\
\hline EGFP-C1 & ACD & Cat No. 400281 \\
\hline Cre-C1 & ACD & Cat No. 402551 \\
\hline Rac1-C1 & ACD & Cat No. 517461 \\
\hline Cx3cr1-C2 & ACD & Cat No. 314221-C2 \\
\hline TLR2-C1 & ACD & Cat No. 317521 \\
\hline Experimental Models: Organisms/Strains & & \\
\hline Cx3cr1-CreERT2 & The Jackson & Strain: 021160 \\
\hline Deposited data & Laboratory & \\
\hline Raw and processed NGS data & & GSE169019 \\
\hline Software and algorithms & Expression Omnibus & \\
\hline Imaris 9.3.1 & Bitplane & \\
\hline SPSS 20.0 & IBM & \\
\hline & & \\
\hline
\end{tabular}

\section{Resource availability}

Further information and requests for resources and reagents should be directed to and will be fulfilled by corresponding author, lanma@fudan.edu.cn and ffwang@fudan.edu.cn.

\section{EXPERIMENTAL MODEL AND SUBJECT DETAILS}

\section{Animals}

Cx3cr1-CreERT2 mice (RRID:IMSR_JAX:021160)(Parkhurst et al., 2013) were purchased from Jackson Laboratory (Sacramento, CA, USA), and were bred to C57BL/6 J for more than 6 generations. C57BL/6J male mice were obtained from the Shanghai Laboratory Animal Center (CAS, 
Shanghai, China) and housed in reverse light-dark cycle (lights-off at 8:00 a.m., lights-on at 8:00 p.m.) with free access to food and water. All experiment procedures for animals were performed strictly in accordance with the National Institutes of Health Guide for the Care and Use of Laboratory Animals, and were approved by the Animal Care and Use Committee of Fudan University.

\section{METHOD DETAILS}

\section{Plasmid construction}

The scramble short hairpin RNA (shRNA) (5'-TCGAAGTATTCCGCGTACGTT-3') and shRNA targeting different region of mouse Atg7 (5'-GCCAACATCCCTGGATACAAG-3' and 5'-TTCTGTCACGGTTCGATAATG-3') were subcloned into the pAAV-CMV-bGlobin-DIO-EGFP-MIR30shRNA vector (Obio Technology, Shanghai, China). Mice Atg7 mRNA coding sequence (NM_001253717.2) tagged with FLAG at the C-terminals was synthesized by GENEWIZ (Shanghai, China), and inserted between NheI and AscI sites of pAAV-Efla-DIO-EYFP to generate pAAV-Efla-DIO-Atg7-Flag. To generate pAAV-Ef1a-DIO-mVenus-PA-Rac1/C450A/T17N, the EYFP in pAAV-Ef1a-DIO-EYFP (Addgene: No. 27056) was replaced with mVenus-PA-Rac1, mVenus-PA-Rac1-C450A and mVenus-PA-Rac1-T17N generated from pTriEx-mVenus-PA-Racl (Addgene: No. 22007), pTriEx-mVenus-PA-Rac1-C450A (Addgene: No. 22021) and pTriEx-mVenus-PA-Rac1-T17N (Addgene: No.22017), respectively. The PA-Racl and PA-Rac1-C450A tagged with HA at the C-terminals were inserted between NheI and AscI sites of $p A A V$-Efla-DIO-EYFP to generate $p A A V-E f 1 a-D I O-P A-R a c 1 / C 450 A-H A$. The RFP-GFP-LC3 coding sequence was obtained from ptfLC3 (Addgene: No.21074) by PCR and inserted between NheI and AscI sites of pAAV-Efla-DIO-EYFP to replace EYFP. 
PA-Rac1-HA,PA-Rac1-C450A-HA and Atg7-Flag obtained by PCR were inserted between NheI and AscI sites of pAAV-Efla-fDIO hChR2(H134R)-EYFP (Addgene: No. 55639) (Fenno et al., 2014a) to generate $p A A V$-EFla-fDIO-PA-Rac1/C450A-HA and pAAV-Efla-fDIO-ATG7-Flag. The EGFP in pAAV-RAM-EGFP (Addgene: No. 84469) (Sorensen et al., 2016) were replaced with Flpo, Cre, or myrmScarlet-I-P2A-post-eGRASP in pAAV-EFla-Flpo (Addgene: No. 55637), pCAG-Cre (Addgene: No. 13775), and pAAV-TIWB-myrmScarlet-I-P2A-post-eGRASP (Addgene: No. 111584) (Choi et al., 2018) to generate pAAV-RAM-Flpo, pAAV-RAM-Cre, and pAAV-RAMmyrmScarlet-I-P2A-post-eGRASP. The mCherry in pLV-PGK-DIO-mCherry-TLR4miR-TLR2miR (Addgene: No.137733) or pLV-PGK-DIO-mCherry-NegmiR (Addgene: No.137732) (Nie et al., 2018) were replaced with EGFP to generate $p L V-P G K-D I O-E G F P \quad-T L R 4 m i R-T L R 2 m i R$ or pLV-PGK-DIO-EGFP -Neg miR. All AAV vectors were packaged by Obio Technology (Shanghai, China) into serotype 2/9, and the Lentiviral vectors were packaged by Genechem Co., Ltd (Shanghai, China).

\section{Surgery}

Mice were anesthetized with $2 \%$ isoflurane during the surgery and were bilaterally injected with 500 $\mathrm{nl}$ of purified AAV $\left(10^{12} \mathrm{IU} / \mathrm{mL}\right)$. The virus was slowly injected into DG (coordinates: $\pm 1.4 \mathrm{~mm}$ mediolateral (ML), $-2.0 \mathrm{~mm}$ anteroposterior (AP), $-1.9 \mathrm{~mm}$ dorsoventral (DV)) with $10 \mu \mathrm{l}$ Hamilton

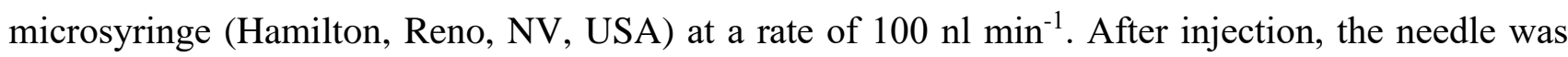
kept in place for 5 min before it was slowly withdrawn. For optical stimulation experiments, $200 \mu \mathrm{m}$ diameter optic fibre (0.37 NA) in the cannula were bilaterally implanted in the DG with an angle of $10^{\circ}$ from the middle to the lateral, and then were secured in place with dental cement. 


\section{Context Fear Conditioning}

Mice were habituated in the experimental room for 3 days, and the diet containing $40 \mathrm{mg} / \mathrm{kg}$ doxycycline (D3447; Sigma-Aldrich, Saint Louis, MO, USA) were taken off 48 hrs before the contextual fear conditioning. Mice were placed in the conditioning chamber illuminated with white light (Med-Associates, St. Albans, VT, USA) and housed in sound attenuating chambers. Fear conditioning was conducted in Context A (Plexiglas observation chamber with stainless-steel bars connected to a shock generator on the floor) for $300 \mathrm{~s}$. The mice received three shocks $(2 \mathrm{~s}$ duration, $0.75 \mathrm{~mA}$ ) at $180 \mathrm{~s}, 240 \mathrm{~s}$ and $300 \mathrm{~s}$ (Guo et al., 2018). The chambers were wiped with 75\% alcohol in each test interval. After the conditioning training, mice were kept back on diet containing Dox with or without PLX3397 (S7818, Selleck, Houston, TX, USA, $290 \mathrm{mg} / \mathrm{kg}$ ). 3 days after training, mice were placed in the training context (context A) and a neutral context (context B, triangular chambers with white plastic floor and grey cover) for $3 \mathrm{~min}$ to record the freezing behavior. For optogenetic activation of Rac1 in vivo, optic fibers were connected to $473 \mathrm{~nm}$ laser diode via a FC/PC adaptor (Newdoon Inc., Hangzhou, China). The laser diode was adjusted to $\sim 20 \mathrm{~mW}$ at the end of the fiber. The light pulse was delivered by light-emitting diode (Newdoon Inc.) at $150 \mathrm{~ms}, 1$ $\mathrm{Hz}$ for $1 \mathrm{hr}$, and the freezing behavior was measured $1 \mathrm{hr}$ after stimulation. Cx3crl-CreER mice were administered with Tamoxifen (T5648, Sigma-Aldrich) dissolved in ethanol and corn oil by intraperitoneal injection $(150 \mathrm{mg} / \mathrm{kg})$ for 3 consecutive days to induce Cre-dependent TLR2/4 miR expression in microglia.

\section{Elevated plus maze (EPM) test}

The elevated plus maze apparatus composed of two open arms and two closed arm $(34.5 \mathrm{~cm}$ length $\times$ 
$6.3 \mathrm{~cm}$ width $\times 19.5 \mathrm{~cm}$ height) were placed $75 \mathrm{~cm}$ above the floor. Mice were allowed to explore EPM freely for 6 min. Mouse behaviors were recorded with an overhead camera. The apparatuses and testing area were wiped with $75 \%$ alcohol in each test interval. Videos were analyzed with TopScan automated detection system (CleverSys. Reston, VA, USA). Percentage of time spent in the open arms and the total number of entries in the open arms were analyszed.

\section{Locomotor Activity Test}

Mice were placed in an open field chamber $(43.2 \mathrm{~cm}$ length $\times 43.2 \mathrm{~cm}$ width $\times 30.5 \mathrm{~cm}$ height, Med-Associates, USA) and allowed to freely explore the space for $30 \mathrm{~min}$. Behavior of mice were monitored with an overhead camera. The overall activity was quantified with TopScan automated detection system (CleverSys, Reston, VA, USA) and the total distance traveled and percentage time spent in the center zone were measured.

\section{Immunofluorescence}

Mice were transcardially perfused with saline followed by $4 \%$ paraformaldehyde (dissolved in $1 \times$ PBS). Brains were isolated and immersed in $4 \%$ PFA at $4{ }^{\circ} \mathrm{C}$ overnight for post fixation, and then the brains were transferred to $30 \%$ sucrose in PBS for 2 days. Brains were frozen and $40-\mu \mathrm{m}$ coronal brain slices were prepared with a cryostat microtome (Leica CM3050S, Nussloch, Germany). For immunostaining, slices were incubated with blocking buffer (PBS containing with 0.3\% Triton X-100 and 10\% normal donkey serum) for $1 \mathrm{hr}$ at room temperature (RT) and then incubated with appropriate primary antibodies at $4{ }^{\circ} \mathrm{C}$ overnight. The primary antibody used are: anti-c-Fos (ab190289, Abcam), anti-Flag (ab205606, Abcam), anti-CD68 (MCA1957, Bio-Rad, California, 
USA), anti-mCherry (M11217, Thermo Fisher, Waltham, MA, USA), anti-RFP (600-401-379, Rockland, Limerick, PA, USA), anti-GFP (600-101-215, Rockland), anti-Iba1 (019-19741, Wako Chemicals, VA, USA). After rinsed in PBS at RT, the slices were incubated with secondary antibodies for $1 \mathrm{hr}$ and then stained with DAPI (10236276001, Roche, Basel-Stadt, Schweiz) for 10 min at RT. The secondary antibodies used are from Jackson ImmunoResearch (West Grove, PA, USA): goat anti-rabbit 488 (111-545-144), goat anti-rabbit Cy3 (111-165-144), donkey anti-rat 647 (712-605-150), goat anti-rat Cy3 (112-165-167). Slices were extensively washed in PBS and mounted onto glass slides in Fluor Mount-G mounting medium (0100-01, Southern Biotech, Birmingham, AL, USA).

\section{Images analysis and quantification}

Fluorescent images were captured by Nikon-A1 confocal microscope (Tokyo, Japan). For analyzing CD68 puncta and immunofluorescent intensity, images of the microglia within $200 \mu \mathrm{m}$ from the optical fiber tips were acquired with a $20 \times$ objective len with $5 \times$ optical zoom and analyzed with open-source software Image J. For analyzing dendritic spines, images were acquired by a $60 \times$ oil objective len with $5 \times$ optical zoom. Z stacks were acquired at $0.5 \mu \mathrm{m}$ interval. Reconstruction and analysis of contact of microglia and the dendrite synaptic structures were carried out by Imaris software (Bitplane, St. Paul, MN, USA). $\mathrm{EGFP}^{+}$Microglia and $\mathrm{mScarlet}^{+}$dendrite and spines were 3D surface rendered with $0.1-\mu \mathrm{m}$ smoothing. The contact areas were calculated with Surface Contact Area XTension (created by Matthew Gastinger).

\section{Microglia isolation, Fluorescence activated cell sorting analysis and RNA}




\section{sequencing}

The mice were perfused with ice-cold $1 \times$ PBS and hippocampi were isolated and incubated with enzyme mix (130-107-677, Miltenyi Biotec, Bergisch Gladbach, Germany) to dissociate into single cell suspension. Debris was removed using debris removal solution (130-107-677, Miltenyi Biotec). Microglia were enriched using CD11b microBeads (130-093-634, Miltenyi Biotec). For fluorescence activated cell sorting analysis, cells were stained with CD11b-PE (12-0112-81, Thermo Fisher) and CD45-FITC antibodies (103108, BioLegend, San Diego, CA, USA) and sorted on CytoFLEX S Flow Cytometer (Beckman Coulter, Brea, CA, USA). For RNA sequencing, RNA in microglia was extracted with Trizol (15596026, Thermo Fisher). Libraries were prepared following the instructions in VAHTS mRNA-seq V3 Library Kit (NR611-01, Vazyme, Nanjing, China) for illumina.

\section{RNA sequencing analysis}

Quality of reads was evaluated using Fastp (Jiang et al., 2021), all samples passed quality control, and reads were aligned to mm10 (GRCm38 from Ensembl) using Hisat2 (Kim et al., 2019). Mapped reads were counted using featureCounts (Wang et al., 2020b) and DESeq2 package (Niu et al., 2018) was used to perform differential gene expression analysis. Gene ontology term enrichment analysis was performed using clusterProfiler (Yu et al., 2012). Gene set enrichment analysis of KEGG were carried out by gseKEGG (part of clusterProfiler) and the results of GSEA were visualized with gseaplot2 (created by Guangchuang Yu). The data have been submitted to NCBI Gene Expression Omnibus (GEO) under accession number GSE169019.

\section{Quantitative RT-PCR}


To downregulate Tlr2/4 in microglia, Cx3crl-CreER mice were bilaterally injected with $L V$-DIO -NegmiR-mCherry or LV-DIO-Tlr2/4miR -mCherry in dorsal dentate gyrus. Two weeks later, the mice were intraperitoneally injected with tamoxifen (T5648, Sigma-Aldrich) at a dose of $150 \mathrm{mg} / \mathrm{kg}$ for 3 consecutive days. Dorsal DG was isolated and the microglia were purified using magnetic-activated cell sorting and mRNA in microglia was extracted with Trizol. cDNA was obtained from total RNA using PrimeScript RT reagent Kit (RR037A, Takara, Shiga, Japan) and then subjected to quantitative RT-PCR with SYBR Premix Ex Taq (RR420A, Takara). The primers used for PCR are as follows: Gapdh forward, 5'-GTGGAGTCATACTGGAACATGTAG-3'; reverse, 5'-AATGGTGAAGGTCGGTGTG-3'; Tlr2 forward, 5'-TGG AATGTCACCAGGCTGC-3'; reverse 5'-GTCCGTGGAAATGGTGGC-3; Tlr4 forward: 5'- ATGGAAAAGCCT CGA ATCCT-3'; reverse, 5'-TCCAAGTTGCCGTTTCTTGT-3'.

\section{single-molecule RNA FISH}

PFA-fixed brains were frozen in OCT and then sliced in $10 \mu \mathrm{m}$ thick coronal sections mounted on a Colorfrost Plus slides (Thermo Scientific) followed by air-dry at room temperature (RT) overnight. Slices were performed target-retrieval and proteolysis using RNAscope ${ }^{\circledR} 2.5$ Universal Pretreatment Reagents (ACD: 322380) In brief, the sections were incubated with hydrogen peroxide for 10 min at RT and proteolysis for $30 \mathrm{~min}$ at $40{ }^{\circ} \mathrm{C}$. SmFISH probes for genes examined: Tlr2-C1 (ACD: 317521), Tlr4-C1 (ACD: 316801), Cx3cr1-C2 (ACD: 314221-C2), Rac1-C1 (ACD: 517461), ATG7-C2 (ACD: 561261-C2), EGFP-C2 (ACD:400281), EGFP-C1 (ACD:400281), Cre-C1 (ACD:402551). The slices were hybridized at $40{ }^{\circ} \mathrm{C}$ for $2 \mathrm{hrs}$. Subsequent amplify signal steps were performed using the RNAscope Multiplex Fluorescent Reagent Kit v2 (ACD: 323110). 


\section{Statistical analysis}

Data were analysed with SPSS 22 software (IBM, Armonk, NY, USA), and plotted by Graphpad Prism. Our sample sizes were based on our previous research (Jiang et al., 2021; Niu et al., 2018; Wang et al., 2020b). The normality test and homogeneity of variance test were performed by the Shapiro-Wilk test and Levene's test. Tukey's multiple comparisons test were performed after student's t test (Unpaired, two tailed), or one-factor analysis of variance (ANOVA) followed. Bonferroni's post hoc analysis was performed after repeated-measures (RM) ANOVA. Data that does not fit a normal distribution were analyzed with a nonparametric test. Two-sample Kolmogorov Smirnov test was used for cumulative frequency plot analysis. Statistical significance was represented as $* \mathrm{P}<0.05 ; * * \mathrm{P}<0.01$, and $* * * \mathrm{P}<0.001$. All data are presented as mean $\pm \mathrm{SEM}$. 


\section{References}

Barrientos, R.M., Frank, M.G., Watkins, L.R., and Maier, S.F. (2010). Memory impairments in healthy aging: Role of aging-induced microglial sensitization. Aging and disease 1, 212-231.

Bordi, M., Berg, M.J., Mohan, P.S., Peterhoff, C.M., Alldred, M.J., Che, S., Ginsberg, S.D., and Nixon, R.A. (2016).

Autophagy flux in CA1 neurons of Alzheimer hippocampus: Increased induction overburdens failing lysosomes to propel neuritic dystrophy. Autophagy 12, 2467-2483. 10.1080/15548627.2016.1239003.

Cangalaya, C., Stoyanov, S., Fischer, K.D., and Dityatev, A. (2020). Light-induced engagement of microglia to focally remodel synapses in the adult brain. eLife 9. 10.7554/eLife.58435.

Castillo, K., Valenzuela, V., Matus, S., Nassif, M., Onate, M., Fuentealba, Y., Encina, G., Irrazabal, T., Parsons, G., Court, F.A., et al. (2013). Measurement of autophagy flux in the nervous system in vivo. Cell death \& disease 4, e917.

10.1038/cddis.2013.421.

Cheadle, L., Rivera, S.A., Phelps, J.S., Ennis, K.A., Stevens, B., Burkly, L.C., Lee, W.A., and Greenberg, M.E. (2020a). Sensory Experience Engages Microglia to Shape Neural Connectivity through a Non-Phagocytic Mechanism. Neuron. 10.1016/j.neuron.2020.08.002.

Cheadle, L., Rivera, S.A., Phelps, J.S., Ennis, K.A., Stevens, B., Burkly, L.C., Lee, W.C.A., and Greenberg, M.E. (2020b). Sensory Experience Engages Microglia to Shape Neural Connectivity through a Non-Phagocytic Mechanism. Neuron 108, $451-+$

Choi, I., Zhang, Y., Seegobin, S.P., Pruvost, M., Wang, Q., Purtell, K., Zhang, B., and Yue, Z. (2020). Microglia clear neuron-released alpha-synuclein via selective autophagy and prevent neurodegeneration. Nat Commun 11, 1386. 10.1038/s41467-020-15119-w.

Choi, J.H., Sim, S.E., Kim, J.I., Choi, D.I., Oh, J., Ye, S., Lee, J., Kim, T., Ko, H.G., Lim, C.S., and Kaang, B.K. (2018). Interregional synaptic maps among engram cells underlie memory formation. Science 360, 430-435.

10.1126/science.aas9204.

Cong, Q., Soteros, B.M., Wollet, M., Kim, J.H., and Sia, G.M. (2020). The endogenous neuronal complement inhibitor SRPX2 protects against complement-mediated synapse elimination during development. Nature neuroscience 23, 1067-1078. 10.1038/s41593-020-0672-0.

Dupont, N., Jiang, S., Pilli, M., Ornatowski, W., Bhattacharya, D., and Deretic, V. (2011). Autophagy-based unconventional secretory pathway for extracellular delivery of IL-1beta. The EMBO journal 30, 4701-4711. 10.1038/emboj.2011.398.

Fenno, L.E., Mattis, J., Ramakrishnan, C., Hyun, M., Lee, S.Y., He, M., Tucciarone, J., Selimbeyoglu, A., Berndt, A., Grosenick, L., et al. (2014a). Targeting cells with single vectors using multiple-feature Boolean logic. Nat Methods 11, 763-U116.

Fenno, L.E., Mattis, J., Ramakrishnan, C., Hyun, M., Lee, S.Y., He, M., Tucciarone, J., Selimbeyoglu, A., Berndt, A., Grosenick, L., et al. (2014b). Targeting cells with single vectors using multiple-feature Boolean logic. Nat Methods 11, 763-772. 10.1038/nmeth.2996.

Fernandez-Arjona, M.D.M., Grondona, J.M., Fernandez-Llebrez, P., and Lopez-Avalos, M.D. (2019). Microglial activation by microbial neuraminidase through TLR2 and TLR4 receptors. Journal of neuroinflammation 16, 245.

10.1186/s12974-019-1643-9.

Ferreira, R., Xapelli, S., Santos, T., Silva, A.P., Cristovao, A., Cortes, L., and Malva, J.O. (2010). Neuropeptide Y modulation of interleukin-1\{beta\} (IL-1\{beta\})-induced nitric oxide production in microglia. The Journal of biological chemistry 285 , 41921-41934. 10.1074/jbc.M110.164020.

Fiebich, B.L., Batista, C.R.A., Saliba, S.W., Yousif, N.M., and de Oliveira, A.C.P. (2018). Role of Microglia TLRs in Neurodegeneration. Frontiers in cellular neuroscience 12, 329. 10.3389/fncel.2018.00329.

Fuhrmann, M., Bittner, T., Jung, C.K., Burgold, S., Page, R.M., Mitteregger, G., Haass, C., LaFerla, F.M., Kretzschmar, H., and Herms, J. (2010). Microglial Cx3cr1 knockout prevents neuron loss in a mouse model of Alzheimer's disease. Nature 
neuroscience 13, 411-413. 10.1038/nn.2511.

Glatigny, M., Moriceau, S., Rivagorda, M., Ramos-Brossier, M., Nascimbeni, A.C., Lante, F., Shanley, M.R., Boudarene, N., Rousseaud, A., Friedman, A.K., et al. (2019). Autophagy Is Required for Memory Formation and Reverses Age-Related Memory Decline. Curr Biol 29, 435-+.

Glick, D., Barth, S., and Macleod, K.F. (2010). Autophagy: cellular and molecular mechanisms. The Journal of pathology 221, 3-12. 10.1002/path.2697.

Gonzalez, C.D., Resnik, R., and Vaccaro, M.I. (2020). Secretory Autophagy and Its Relevance in Metabolic and Degenerative Disease. Frontiers in endocrinology 11, 266. 10.3389/fendo.2020.00266.

Guo, N., Soden, M.E., Herber, C., Kim, M.T., Besnard, A., Lin, P., Ma, X., Cepko, C.L., Zweifel, L.S., and Sahay, A. (2018). Dentate granule cell recruitment of feedforward inhibition governs engram maintenance and remote memory generalization. Nature medicine 24, 438-449. 10.1038/nm.4491.

Hammond, T.R., Marsh, S.E., and Stevens, B. (2019). Immune Signaling in Neurodegeneration. Immunity 50, $955-974$. 10.1016/j.immuni.2019.03.016.

Heavner, W.E., and Smith, S.E.P. (2020). Resolving the Synaptic versus Developmental Dichotomy of Autism Risk Genes. Trends in neurosciences 43, 227-241. 10.1016/j.tins.2020.01.009.

Holtmaat, A., and Caroni, P. (2016). Functional and structural underpinnings of neuronal assembly formation in learning. Nature neuroscience 19, 1553-1562. 10.1038/nn.4418.

Hong, S., Beja-Glasser, V.F., Nfonoyim, B.M., Frouin, A., Li, S., Ramakrishnan, S., Merry, K.M., Shi, Q., Rosenthal, A., Barres, B.A., et al. (2016). Complement and microglia mediate early synapse loss in Alzheimer mouse models. Science 352, 712-716. 10.1126/science.aad8373.

Hui, K.K., Takashima, N., Watanabe, A., Chater, T.E., Matsukawa, H., Nekooki-Machida, Y., Nilsson, P., Endo, R., Goda, Y., Saido, T.C., et al. (2019). GABARAPs dysfunction by autophagy deficiency in adolescent brain impairs GABAA receptor trafficking and social behavior. Science advances 5, eaau8237. 10.1126/sciadv.aau8237.

Jiang, C., Wang, X., Le, Q., Liu, P., Liu, C., Wang, Z., He, G., Zheng, P., Wang, F., and Ma, L. (2021). Morphine coordinates SST and PV interneurons in the prelimbic cortex to disinhibit pyramidal neurons and enhance reward. Molecular psychiatry 26, 1178-1193. 10.1038/s41380-019-0480-7.

Joshi, G., Gan, K.A., Johnson, D.A., and Johnson, J.A. (2015). Increased Alzheimer's disease-like pathology in the APP/PS1 Delta E9 mouse model lacking Nrf2 through modulation of autophagy. Neurobiol Aging 36, 664-679.

Kawasaki, T., and Kawai, T. (2014). Toll-like receptor signaling pathways. Frontiers in immunology 5, 461. 10.3389/fimmu.2014.00461.

Kim, C., Ho, D.H., Suk, J.E., You, S., Michael, S., Kang, J., Joong Lee, S., Masliah, E., Hwang, D., Lee, H.J., and Lee, S.J. (2013). Neuron-released oligomeric alpha-synuclein is an endogenous agonist of TLR2 for paracrine activation of microglia. Nat Commun 4, 1562. 10.1038/ncomms2534.

Kim, D., Paggi, J.M., Park, C., Bennett, C., and Salzberg, S.L. (2019). Graph-based genome alignment and genotyping with HISAT2 and HISAT-genotype. Nature biotechnology 37, 907-915. 10.1038/s41587-019-0201-4.

Kim, H.J., Cho, M.H., Shim, W.H., Kim, J.K., Jeon, E.Y., Kim, D.H., and Yoon, S.Y. (2017). Deficient autophagy in microglia impairs synaptic pruning and causes social behavioral defects. Molecular psychiatry 22, 1576-1584.

10.1038/mp.2016.103.

Krasnow, S.M., Knoll, J.G., Verghese, S.C., Levasseur, P.R., and Marks, D.L. (2017). Amplification and propagation of interleukin-1beta signaling by murine brain endothelial and glial cells. Journal of neuroinflammation 14, 133. 10.1186/s12974-017-0908-4.

Kulkarni, A., Chen, J., and Maday, S. (2018). Neuronal autophagy and intercellular regulation of homeostasis in the brain. Current opinion in neurobiology 51, 29-36. 10.1016/j.conb.2018.02.008.

Lachance, V., Wang, Q., Sweet, E., Choi, I., Cai, C.Z., Zhuang, X.X., Zhang, Y., Jiang, J.L., Blitzer, R.D., Bozdagi-Gunal, O., et 
al. (2019). Autophagy protein NRBF2 has reduced expression in Alzheimer's brains and modulates memory and amyloid-beta homeostasis in mice. Molecular neurodegeneration 14, 43. 10.1186/s13024-019-0342-4.

Lewitus, G.M., Konefal, S.C., Greenhalgh, A.D., Pribiag, H., Augereau, K., and Stellwagen, D. (2016). Microglial TNF-alpha Suppresses Cocaine-Induced Plasticity and Behavioral Sensitization. Neuron 90, 483-491.

10.1016/j.neuron.2016.03.030.

Liao, K., Guo, M., Niu, F., Yang, L., Callen, S.E., and Buch, S. (2016). Cocaine-mediated induction of microglial activation involves the ER stress-TLR2 axis. Journal of neuroinflammation 13, 33. 10.1186/s12974-016-0501-2.

Lipinski, M.M., Zheng, B., Lu, T., Yan, Z., Py, B.F., Ng, A., Xavier, R.J., Li, C., Yankner, B.A., Scherzer, C.R., and Yuan, J. (2010). Genome-wide analysis reveals mechanisms modulating autophagy in normal brain aging and in Alzheimer's disease. Proceedings of the National Academy of Sciences of the United States of America 107, 14164-14169.

10.1073/pnas.1009485107.

Liu, Y., Dai, Y., Li, Q., Chen, C., Chen, H., Song, Y., Hua, F., and Zhang, Z. (2020). Beta-amyloid activates NLRP3 inflammasome via TLR4 in mouse microglia. Neuroscience letters 736, 135279. 10.1016/j.neulet.2020.135279.

Lui, H., Zhang, J., Makinson, S.R., Cahill, M.K., Kelley, K.W., Huang, H.Y., Shang, Y., Oldham, M.C., Martens, L.H., Gao, F., et al. (2016). Progranulin Deficiency Promotes Circuit-Specific Synaptic Pruning by Microglia via Complement Activation. Cell 165, 921-935. 10.1016/j.cell.2016.04.001.

Lv, L., Liu, Y., Xie, J., Wu, Y., Zhao, J., Li, Q., and Zhong, Y. (2019). Interplay between alpha2-chimaerin and Rac1 activity determines dynamic maintenance of long-term memory. Nat Commun 10, 5313. 10.1038/s41467-019-13236-9. Mancias, J.D., and Kimmelman, A.C. (2016). Mechanisms of Selective Autophagy in Normal Physiology and Cancer. Journal of molecular biology 428, 1659-1680. 10.1016/j.jmb.2016.02.027.

Miyamoto, A., Wake, H., Ishikawa, A.W., Eto, K., Shibata, K., Murakoshi, H., Koizumi, S., Moorhouse, A.J., Yoshimura, Y., and Nabekura, J. (2016). Microglia contact induces synapse formation in developing somatosensory cortex. Nat Commun 7, 12540. 10.1038/ncomms12540.

Nguyen, P.T., Dorman, L.C., Pan, S., Vainchtein, I.D., Han, R.T., Nakao-Inoue, H., Taloma, S.E., Barron, J.J., Molofsky, A.B., Kheirbek, M.A., and Molofsky, A.V. (2020). Microglial Remodeling of the Extracellular Matrix Promotes Synapse Plasticity. Cell 182, 388-403 e315. 10.1016/j.cell.2020.05.050.

Nie, X., Kitaoka, S., Tanaka, K., Segi-Nishida, E., Imoto, Y., Ogawa, A., Nakano, F., Tomohiro, A., Nakayama, K., Taniguchi, M., et al. (2018). The Innate Immune Receptors TLR2/4 Mediate Repeated Social Defeat Stress-Induced Social Avoidance through Prefrontal Microglial Activation. Neuron 99, 464-479 e467. 10.1016/j.neuron.2018.06.035.

Niu, B., Liu, P., Shen, M., Liu, C., Wang, L., Wang, F., and Ma, L. (2018). GRK5 Regulates Social Behavior Via Suppression of mTORC1 Signaling in Medial Prefrontal Cortex. Cerebral cortex 28, 421-432. 10.1093/cercor/bhw364.

Osimo, E.F., Beck, K., Reis Marques, T., and Howes, O.D. (2019). Synaptic loss in schizophrenia: a meta-analysis and systematic review of synaptic protein and mRNA measures. Molecular psychiatry 24, 549-561.

10.1038/s41380-018-0041-5.

Parkhurst, C.N., Yang, G., Ninan, I., Savas, J.N., Yates, J.R., Lafaille, J.J., Hempstead, B.L., Littman, D.R., and Gan, W.B. (2013). Microglia Promote Learning-Dependent Synapse Formation through Brain-Derived Neurotrophic Factor. Cell 155, 1596-1609.

Rajendran, L., and Paolicelli, R.C. (2018). Microglia-Mediated Synapse Loss in Alzheimer's Disease. The Journal of neuroscience : the official journal of the Society for Neuroscience 38, 2911-2919. 10.1523/JNEUROSCI.1136-17.2017.

Sarraf, S.A., Raman, M., Guarani-Pereira, V., Sowa, M.E., Huttlin, E.L., Gygi, S.P., and Harper, J.W. (2013). Landscape of the PARKIN-dependent ubiquitylome in response to mitochondrial depolarization. Nature 496, 372-376.

10.1038/nature12043.

Schafer, D.P., Lehrman, E.K., Kautzman, A.G., Koyama, R., Mardinly, A.R., Yamasaki, R., Ransohoff, R.M., Greenberg, M.E., Barres, B.A., and Stevens, B. (2012). Microglia sculpt postnatal neural circuits in an activity and complement-dependent 
manner. Neuron 74, 691-705. 10.1016/j.neuron.2012.03.026.

Sellgren, C.M., Gracias, J., Watmuff, B., Biag, J.D., Thanos, J.M., Whittredge, P.B., Fu, T., Worringer, K., Brown, H.E., Wang, J., et al. (2019). Increased synapse elimination by microglia in schizophrenia patient-derived models of synaptic pruning. Nature neuroscience 22, 374-385. 10.1038/s41593-018-0334-7.

Shi, Q., Colodner, K.J., Matousek, S.B., Merry, K., Hong, S., Kenison, J.E., Frost, J.L., Le, K.X., Li, S., Dodart, J.C., et al. (2015). Complement C3-Deficient Mice Fail to Display Age-Related Hippocampal Decline. The Journal of neuroscience : the official journal of the Society for Neuroscience 35, 13029-13042. 10.1523/JNEUROSCI.1698-15.2015.

Sorensen, A.T., Cooper, Y.A., Baratta, M.V., Weng, F.J., Zhang, Y., Ramamoorthi, K., Fropf, R., LaVerriere, E., Xue, J., Young, A., et al. (2016). A robust activity marking system for exploring active neuronal ensembles. eLife 5. 10.7554/eLife.13918. Spangenberg, E.E., Lee, R.J., Najafi, A.R., Rice, R.A., Elmore, M.R., Blurton-Jones, M., West, B.L., and Green, K.N. (2016). Eliminating microglia in Alzheimer's mice prevents neuronal loss without modulating amyloid-beta pathology. Brain : a journal of neurology 139, 1265-1281. 10.1093/brain/aww016.

Spittau, B. (2017). Aging Microglia-Phenotypes, Functions and Implications for Age-Related Neurodegenerative Diseases. Frontiers in aging neuroscience 9, 194. 10.3389/fnagi.2017.00194.

Stirling, D.P., Cummins, K., Mishra, M., Teo, W., Yong, V.W., and Stys, P. (2014). Toll-like receptor 2-mediated alternative activation of microglia is protective after spinal cord injury. Brain : a journal of neurology 137, 707-723.

10.1093/brain/awt341.

Stowell, R.D., Sipe, G.O., Dawes, R.P., Batchelor, H.N., Lordy, K.A., Whitelaw, B.S., Stoessel, M.B., Bidlack, J.M., Brown, E., Sur, M., and Majewska, A.K. (2019). Noradrenergic signaling in the wakeful state inhibits microglial surveillance and synaptic plasticity in the mouse visual cortex. Nature neuroscience 22, 1782-1792. 10.1038/s41593-019-0514-0.

Su, F., Bai, F., Zhou, H., and Zhang, Z. (2016). Microglial toll-like receptors and Alzheimer's disease. Brain, behavior, and immunity 52, 187-198. 10.1016/j.bbi.2015.10.010.

Todd, L., Palazzo, I., Suarez, L., Liu, X., Volkov, L., Hoang, T.V., Campbell, W.A., Blackshaw, S., Quan, N., and Fischer, A.J. (2019). Reactive microglia and IL1beta/IL-1R1-signaling mediate neuroprotection in excitotoxin-damaged mouse retina. Journal of neuroinflammation 16, 118. 10.1186/s12974-019-1505-5.

Wang, C., Yue, H., Hu, Z., Shen, Y., Ma, J., Li, J., Wang, X.D., Wang, L., Sun, B., Shi, P., et al. (2020a). Microglia mediate forgetting via complement-dependent synaptic elimination. Science 367, 688-694. 10.1126/science.aaz2288.

Wang, Z., Jin, T., Le, Q., Liu, C., Wang, X., Wang, F., and Ma, L. (2020b). Retrieval-Driven Hippocampal NPTX2 Plasticity Facilitates the Extinction of Cocaine-Associated Context Memory. Biological psychiatry 87, 979-991.

10.1016/j.biopsych.2019.10.009.

Weinhard, L., di Bartolomei, G., Bolasco, G., Machado, P., Schieber, N.L., Neniskyte, U., Exiga, M., Vadisiute, A., Raggioli, A., Schertel, A., et al. (2018). Microglia remodel synapses by presynaptic trogocytosis and spine head filopodia induction. Nat Commun 9, 1228. 10.1038/s41467-018-03566-5.

Werneburg, S., Feinberg, P.A., Johnson, K.M., and Schafer, D.P. (2017). A microglia-cytokine axis to modulate synaptic connectivity and function. Current opinion in neurobiology 47, 138-145. 10.1016/j.conb.2017.10.002.

Wu, W., Du, S., Shi, W., Liu, Y., Hu, Y., Xie, Z., Yao, X., Liu, Z., Ma, W., Xu, L., et al. (2019). Inhibition of Rac1-dependent forgetting alleviates memory deficits in animal models of Alzheimer's disease. Protein \& cell 10, 745-759.

10.1007/s13238-019-0641-0.

Wu, Y., Dissing-Olesen, L., MacVicar, B.A., and Stevens, B. (2015). Microglia: Dynamic Mediators of Synapse Development and Plasticity. Trends in immunology 36, 605-613. 10.1016/j.it.2015.08.008.

Yao, L., Kan, E.M., Lu, J., Hao, A., Dheen, S.T., Kaur, C., and Ling, E.A. (2013). Toll-like receptor 4 mediates microglial activation and production of inflammatory mediators in neonatal rat brain following hypoxia: role of TLR4 in hypoxic microglia. Journal of neuroinflammation 10, 23. 10.1186/1742-2094-10-23.

Yu, G.C., Wang, L.G., Han, Y.Y., and He, Q.Y. (2012). clusterProfiler: an R Package for Comparing Biological Themes Among 
Gene Clusters. Omics 16, 284-287.

Zhan, Y., Paolicelli, R.C., Sforazzini, F., Weinhard, L., Bolasco, G., Pagani, F., Vyssotski, A.L., Bifone, A., Gozzi, A., Ragozzino, D., and Gross, C.T. (2014). Deficient neuron-microglia signaling results in impaired functional brain connectivity and social behavior. Nature neuroscience 17, 400-406. 10.1038/nn.3641.

Zhao, J., Bi, W., Zhang, J., Xiao, S., Zhou, R., Tsang, C.K., Lu, D., and Zhu, L. (2020). USP8 protects against lipopolysaccharide-induced cognitive and motor deficits by modulating microglia phenotypes through TLR4/MyD88/NF-kappaB signaling pathway in mice. Brain, behavior, and immunity 88, 582-596.

10.1016/j.bbi.2020.04.052.

Zhou, Y.Y., Li, Y., Jiang, W.Q., and Zhou, L.F. (2015). MAPK/JNK signalling: a potential autophagy regulation pathway. Bioscience reports 35. 10.1042/BSR20140141. 
Figure 1. Increased ATG7 in DG engrams impaired fear memory retrieval and microglia activation.

(A) Schematic of the experimental procedure for labeling DG engrams. (B) Representative confocal images and quantitative analysis of $\mathrm{EGFP}^{+}$cell number and the percentage of $\mathrm{EGFP}^{+} \mathrm{c}-\mathrm{Fos}^{+}$cells following exposure to context A. Green: EGFP; Red: c-Fos; Blue: DAPI. Scale bar: $50 \mu \mathrm{m}$. White arrows indicates the $\mathrm{EGFP}^{+} \mathrm{c}-\mathrm{Fos}^{+}$cells. $\mathrm{EGFP}^{+} / \mathrm{mm}^{2}$ : $\mathrm{t}=2.892, \mathrm{P}=0.034 ; \% \mathrm{c}-\mathrm{Fos}^{+} \mathrm{EGFP}^{+} / \mathrm{EGFP}^{+}$: $\mathrm{t}=3.002, \mathrm{P}=0.03$, Two-tailed unpaired t-test. (C-D) Representative confocal images (C) and quantification (D) of the smFish against Atg7 mRNA in $\mathrm{EGFP}^{+}$cell. Green: Egfp; Red: Atg7; Blue: DAPI. Scale bar: $20 \mu \mathrm{m} . \mathrm{Z}=7.285$, P<0.001, Kolmogorov-Smirnov (KS) test. $(\mathrm{E}-\mathrm{H})$ Mice were infected with AAV-RAM-Cre, AAV-DIO -Scramble-shRNA-EGFP or AAV-DIO-Atg7-shRNA-1-EGFP or AAV-DIO-Atg7-shRNA-2-EGFP bilaterally in dorsal DG. (E) Experimental procedure to investigate the effect of ATG7 knockdown in the DG engrams on the retrieval of contextual fear memory. (F) Representative confocal images and quantification of smFish against $\operatorname{Atg} 7$ and Egfp mRNA to assess the knockdown efficiency of $\operatorname{Atg} 7-\operatorname{shRNA}$ within $\mathrm{EGFP}^{+}$ engrams. Green: Egfp; Red: Atg7; Blue: DAPI. Scale bar: $50 \mu \mathrm{m} .(\mathrm{G})$ Freezing level of the mice during fear conditioning. F Freatment $\times$ trial $(6,93)=0.644, \mathrm{P}=0.695$, RM ANOVA. (H) Freezing level 3 days after fear conditioning in context A and neutral context (context B). $\mathrm{F}_{\text {treatment } \times \text { context }(2,31)}=2.330$, $\mathrm{P}=0.114$, Repeated measurement ANOVA. Context A: Scramble versus Atg7sh-1. $\mathrm{P}=0.006 ;$ Scramble versus Atg7sh-2, $\mathrm{P}=0.002$; Bonferroni post hoc test. (I) Experimental procedure to overexpressing Atg7 in DG engrams and pharmacological depletion of microglia on the retrieval of contextual fear memory. Mice were fed with food containing Dox (control) or food containing Dox and PLX3329 after engrams labeling. (J) Representative confocal images of ATG7 or EYFP expression in Cre 
regions. Green: EYFP/ATG7-Flag; Red: Cre; Blue: DAPI. Scale bar:50 $\mu \mathrm{m}$. (K) Representative images and quantification of CD68 puncta within Iba1 microglia. Red: Iba1; Blue: CD68. Scale bar: $10 \mu \mathrm{m} . \mathrm{t}=-7.968, \mathrm{P}<0.001$, Two-tailed unpaired t-test. (L-M) Representative confocal images (L) and quantification (M) of $\mathrm{Iba1}^{+}$microglia number in the DG. Red: Iba1; Blue: DAPI. Scale bar:100 $\mu \mathrm{m} . \mathrm{t}=4.308, \mathrm{P}=0.005$, Two-tailed unpaired t-test. (N) Freezing level of mice during fear conditioning. $\chi 2=3.650, \mathrm{P}=0.161$, Friedman's $\mathrm{M}$ test. $(\mathrm{O})$ Freezing level in context $\mathrm{A}$ and context $\mathrm{B} . \mathrm{F}_{\text {treatment } \times \text { context }}$ $(2,45)=5.406, \mathrm{P}=0.008, \mathrm{RM}$ ANOVA. Context A: EGFP versus ATG7-Flag, $\mathrm{P}<0.001$; ATG7-Flag versus $A T G 7-F l a g+P L X, P=0.003$. Bonferroni post $\mathrm{t}$ test. Data are presented as mean $\pm \mathrm{SEM} .{ }^{*} P<$ $0.05, * * P<0.01, * * * P<0.001$. 
Figure 2. Upregulation of ATG7 within DG engrams induces immune response and upregulates the expression of Tlr2/4 within DG microglia.

(A) Experiment procedure. Dorsal DG bilaterally infected with AAV-RAM-Cre mixed with either $A A V$-DIO-EGFP or AAV-DIO-Atg7-Flag was dissected 3 days after fear conditioning, and the microglia were isolated by magnetic-activated cell sorting (MACs). (B) Fluorescence-activated cell sorting (FACS) analysis in Blank-Ctrl group (without antibody) and Anti-CD45/CD11b group (with anti-CD45/CD11b antibody) after MACs. (C-D) mRNA of microglia was extracted and subjected to RNA-Seq. Heat map of differentially expressed genes of the microglia(C) and the Gene ontology (GO) analysis of upregulated genes in microglia (D) in the groups overexpressing EYFP or ATG7 within DG engrams. (E) GSEA results of the signature genes of Toll-like receptor (TLR) signaling pathway in the microglia when overexpressing ATG7 within DG engrams. (F) The heat map expression of the changed genes involved in immune response. (G) GSEA results of the signature genes of TNF signaling pathway and cytokine-cytokine receptor interaction in the microglia when overexpressing ATG7 within DG engrams. (H) Experiment procedure to test of the level of Tlr2/4 mRNA in DG (I-K) Representative images (I) and quantification (J-K) of the smFish against Tlr2/4 mRNA in DG microglia $\left(\mathrm{Cx} 3 \mathrm{cr} \mathrm{l}^{+}\right.$cells) when overexpressing EGFP or ATG7-Flag within the DG engrams. Green: Cx3cr1; Red: Tlr2/4; Blue: DAPI. Scale bar: $50 \mu \mathrm{m}$. Tlr2: Z=5.695, P<0.001; Tlr4: $\mathrm{Z}=2.381, \mathrm{P}<0.001, \mathrm{KS}$ test. Data are presented as mean $\pm \mathrm{SEM} . * * * P<0.001$. 
Figure 3. TLR2/4 in DG microglia mediates ATG7-induced spine elimination of the DG engrams.

(A) Cx3cr1-CreER mice infected with $L V$-DIO -NegmiR-mCherry or LV-DIO-Tlr2/4miR-mCherry in dorsal DG was administered with TAM 1 day after engrams labeling to downregulate $T l r 2 / 4$ in DG microglia. Microglia in the DG were isolated using MACs, and the mRNA was extracted and subjected to qRT-PCR to quantify the mRNA level of Tlr2/4 in the microglia. Tlr2: $\mathrm{t}=3.794, \mathrm{P}=0.013$; Tlr4: $\mathrm{t}=3.631, \mathrm{P}=0.015$. Two-tailed unpaired $\mathrm{t}$-test. (B) Experiment procedure to test the effect of Tlr2/4 knockdown in DG microglia on the retrieval of contextual fear memory. (C) Representative confocal images of the ATG7 and Tlr2/4miR-mCherry expression. Green: ATG7-Flag; Red: mCherry; Blue: DAPI. Scale bar:100 $\mu \mathrm{m}$. (D) Freezing level of Cx3crl-CreER mice during the fear conditioning. $\chi 2=2.070, \mathrm{P}=0.355$, Friedman's $\mathrm{M}$ test. (E) Freezing level of $C x 3 \mathrm{cr} 1-\mathrm{CreER}$ mice 3 days after conditioning in context $\mathrm{A}$ and context $\mathrm{B}$. $\mathrm{F}_{\text {treatment } \mathrm{x} \text { context }(2,25)}=8.517, \mathrm{P}=0.002, \mathrm{RM}$ ANOVA. Context A: EYFP+NegmiR versus ATG7-Flag + NegmiR: $\mathrm{P}<0.001 ;$ ATG7-Flag + NegmiR versus ATG7-Flag + Tlr2/4miR: $\mathrm{P}<0.001$. Bonferroni post $\mathrm{t}$ test. (F) Experiment procedure to assess autophagy-driven synaptic elimination. (G) Representative 3D reconstruction of the interaction of microglia and dendrites of the engrams within DG. (H) Quantification of microglia-dendrite contact area in the DG of Cx3crl-CreER mice. Green: EGFP; Red: mScarlet; Scale bar :5 $\mu$ m. (I-J) Representative cofocal images (I) and quantification (J) of spine density within DG engrams of Cx3cr1-CreER mice. $\mathrm{F}=22.418, \mathrm{P}<0.001$, One-way ANOVA. EGFP + NegmiR versus ATG7-Flag+ NegmiR: $\mathrm{P}<0.001$; ATG7-Flag+ NegmiR versus ATG7-Flag+Tlr2/4miR: $\mathrm{P}<0.001$. Bonferroni post $\mathrm{t}$ test. Red: biocytin; Scale bar :5 $\mu \mathrm{m}$. Data are presented as mean \pm SEM. $* P<0.05, * * * P<0.001$. 
Figure 4. Rac1 within DG engram is upregulated in aged mice and leads to the impairment of contexture fear memory retrieval and the enhancement of autophagy within the engrams.

(A-B) Representative images (A) and quantification (B) of the smFish against Racl in Egfp ${ }^{+}$cell. $\mathrm{Z}=6.873, \mathrm{P}<0.001, \mathrm{KS}$ test. Green: Egfp; Red: Rac1; Blue: DAPI. Scale bar:50 $\mu \mathrm{m}$. (C-D) Schematic of optical activated Rac1 system and the experimental procedure to optical activation of Rac1 within dorsal DG engrams on the retrieval of contexture fear memory. 3 days after engrams labeling, the $473 \mathrm{~nm}$ laser pulse was delivered in the DG for $150 \mathrm{~ms}$ at $1 \mathrm{~Hz}$ for $1 \mathrm{hr}$, and the freezing level in context A was measured $1 \mathrm{hr}$ after optical stimulation. (E-F) Brains were collected $1.5 \mathrm{hrs}$ after re-exposure to context A for c-Fos staining. Representative images (E) and quantification of the percentage of $\mathrm{c}-F o s^{+}$components in $\mathrm{EGFP}^{+}$engrams $(\mathrm{F}) . \mathrm{t}=3.112, \mathrm{P}=0.041$, Two-tailed unpaired t-test. Green:C450A/Pa Rac1; Red: c-Fos; Blue: DAPI. Scale bar: $50 \mu \mathrm{m}$. (G) Freezing level during fear conditioning. $\chi 2=0.03, \mathrm{P}=0.861$, Friedman's M test. $(\mathrm{H})$ Freezing level in context $\mathrm{A}$ and context B $1 \mathrm{hr}$ after optical stimulation. $\mathrm{F}_{\text {treatment }} \mathrm{x}$ context $(1,16)=4.531, \mathrm{P}=0.049, \mathrm{RM}$ ANOVA. Context A: C450A versus $\mathrm{Pa}$ Rac1: $\mathrm{P}=0.019$, Bonferroni post t-test. (I) Freezing level in context A and context B 24 hrs after optical stimulation. $F_{\text {treatment } x \text { context }(1,23)}=5.06, \mathrm{P}=0.034, \mathrm{RM}$ ANOVA. Context A: C450A versus Pa Rac1: $\mathrm{P}=0.013$, Bonferroni post t-test. (J) Freezing level during fear conditioning. $\chi 2=2.133, \mathrm{P}=0.144$, Friedman's $\mathrm{M}$ test. $(\mathrm{K})$ Freezing level in context A and context $\mathrm{B} 1 \mathrm{hr}$ after optical stimulation. F Freatment $x$ context $(1,20)=2.672, \mathrm{P}=0.118, \mathrm{RM}$ ANOVA. Context A: C450A versus T17N: $\mathrm{P}=0.006$. Bonferroni post $\mathrm{t}$ test. (L) Freezing level in context A and context B $24 \mathrm{hrs}$ after optical stimulation. $\mathrm{F}_{\text {treatment } \mathrm{x} \text { context }}(1,23)=1.116, \mathrm{P}=0.302$, RM ANOVA. (M) Experimental procedure to assess autophagy after optical activation of Rac1 in the DG engrams. (N-O) Representative confocal images $(\mathrm{N})$ and quantification $(\mathrm{O})$ of autophagy influx. 3 days after the 

made available under aCC-BY 4.0 International license.

engrams labeling, the light pulse was delivered for $150 \mathrm{~ms}$ at $1 \mathrm{~Hz}$ for $1 \mathrm{hr}$ and autophagy influx was measured $1 \mathrm{hr}$ after stimulation. $\mathrm{Z}=-9.287, \mathrm{P}<0.001$, Mann-Whitney U test. Red: RFP; Green: EGFP. Scale bar: $50 \mu \mathrm{m}$. Data are presented as mean \pm SEM. $* P<0.05, * * * P<0.001$ 
Figure 5. Activation of Rac1 within DG engrams promotes the expression of ATG7 and activation of microglia in a TLR2/4-dependent manner.

(A) Experimental procedure to assess the microglia activation after optical activation of Rac1 in DG engrams. (B-C) Representative images (B) and quantification (C) of the smFish against Atg7 mRNA in $\mathrm{C} 450 \mathrm{~A}$ and Pa Rac1 groups. Z=3.872, P<0.001, KS test. Green: Egfp; Red: Atg7; Blue: DAPI. Scale bar: $50 \mu \mathrm{m}$. (D-E) Representative images(D) and quantification (E) of $\mathrm{CD}^{+} 8^{+}$puncta within microglia in $\mathrm{C} 450 \mathrm{~A}$ and $\mathrm{Pa}$ Rac1 group.t=-2.256, $\mathrm{P}=0.027$, Two-tailed unpaired t-test. Green:C450A/Pa Rac1; Red: Iba1; Blue: CD68. Scale bar: left: $100 \mu \mathrm{m}$; right:10 $\mu \mathrm{m}$. (F-H) Representative images (F) and quantification $(\mathrm{G}-\mathrm{H})$ of the smFish against $T l r 2 / 4$ and $C x 3 c r 1^{+}$ mRNA in the DG. Tlr2: $\mathrm{Z}=1.792, \mathrm{P}=0.03 ;$ Tlr4: $\mathrm{Z}=2.583, \mathrm{P}<0.001$. KS test. Green: $C x 3 c r 1$; Red: Tlr2/4; Blue: DAPI. Scale bar: top: $50 \mu \mathrm{m}$; bottom: $50 \mu \mathrm{m}$. (I) Experiment procedure to test the effect of knocking down Tlr2/4 within DG and activating Rac1 within DG engrams on the activation of microglia. (J) Representative images of lentivirus expression in DG microglia. Green: Iba1; Red: mCherry. Scale bar:20 $\mu \mathrm{m}$. (K-L) Representative images (K) and quantifications (L) of CD68+ puncta in mCherry $^{+}$microglia. $\chi 2=8.184, \mathrm{P}=0.017$, Kruskal-Wallis $\mathrm{H}$ test. $\mathrm{C} 450 \mathrm{~A}+\mathrm{NegmiR}$ versus Pa Rac1+NegmiR: $\mathrm{P}=0.013$. Green: $C D 68$; Red: $m$ Cherry; Blue: DAPI. Scale bar: left:50 $\mu$; right: $10 \mu \mathrm{m}$. Data are presented as mean \pm SEM. ${ }^{*} P<0.05, * * P<0.01, * * * P<0.001$. 
Figure 6. ATG7 within DG engram and TLR2/4 within DG microglia mediates the impaired retrieval of contexture fear memory induced by activation of Rac1 in DG engrams.

(A) Experiment procedure to test the effect of Atg7 knockdown and Rac1 activation within DG engrams on the retrieval of contextual fear memory. (B) Freezing level of mice during fear conditioning. $\chi 2=3.146, \mathrm{P}=0.207$, Friedman's $\mathrm{M}$ test. $(\mathrm{C})$ Freezing level of mice in context $\mathrm{A}$ and context B 3 days after fear conditioning. $\mathrm{F}_{\text {treatment } x \text { context }(2,33)}=6.2, \mathrm{P}=0.005, \mathrm{RM}$ ANOVA. Context A: C450A +Scramble versus Pa Rac1 + Scramble: $\mathrm{P}<0.001 ; \mathrm{Pa}$ Rac1 + Scramble versus Pa Rac1 + Atg7sh-2: $\mathrm{P}<0.001$. Bonferroni post $\mathrm{t}$ test. (D) Experiment procedure to test the effect of microglia depletion and activation of Rac1in DG engrams on the retrieval of contextual fear memory. Mice were given control food (only containing dox) or PLX food (containing dox and PLX) after engrams labeling. (E) Freezing level of mice during fear conditioning. $\chi 2=1.319, \mathrm{P}=0.517$, Friedman's $\mathrm{M}$ test. (F) Freezing level of mice 3 days after fear conditioning in context $\mathrm{A}$ and context $\mathrm{B}$. $\mathrm{F}_{\text {treatment } \mathrm{x} \text { context }}$ $(2,26)=6.406, \mathrm{P}=0.005, \mathrm{RM}$ ANOVA. Context A: C450A + Ctrl versus Pa Rac1 + Ctrl: $\mathrm{P}=0.005 ; \mathrm{Pa}$ Rac1 + Ctrl versus Pa Rac1 + PLX: P=0.001. Bonferroni post t test. $(\mathrm{G}-\mathrm{H})$ the effect of microglia activation and Rac1 activation in the DG on locomotor activity $(\mathrm{G})$ and anxiety(H). (I) Experiment procedure to test the effect of Tlr2/4 knockdown in DG microglia and Rac1 activation in the DG engrams on the retrieval of contextual fear memory. (J) Freezing level of Cx3crl-CreER mice during fear conditioning. $\chi 2=3.449, \mathrm{P}=0.178$, Friedman's M test. (K) Freezing level of $C x 3 c r 1-C r e E R$ mice 3 days after fear conditioning in context $\mathrm{A}$ and context $\mathrm{B}$. $\mathrm{F}_{\text {treatment } \mathrm{x} \text { context }(2,22)}=3.214, \mathrm{P}=0.06, \mathrm{RM}$ ANOVA. Context A: C450A +NegmiR versus Pa Rac1 + NegmiR: $\mathrm{P}=0.005 ; \mathrm{Pa} \mathrm{Rac} 1+$ NegmiR versus $\mathrm{Pa}$ Rac1 + Tlr2/4miR: $\mathrm{P}=0.01$. Bonferroni post $\mathrm{t}$ test. (L-M) The effect of optical-activating Rac1 in DG engrams and knocking down Tlr2/4 in DG microglia on locomotor activity (L) and 
bioRxiv preprint doi: https://doi.org/10.1101/2021.08.26.457763; this version posted August 28, 2021. The copyright holder for this preprint (which was not certified by peer review) is the author/funder, who has granted bioRxiv a license to display the preprint in perpetuity. It is made available under aCC-BY 4.0 International license.

anxiety(M) of Cx3cr1-CreER mice. Data are presented as mean \pm SEM. $* P<0.05, * * P<0.01, * * * P$ $<0.001$. 
Figure 7. ATG7-dependent autophagy in DG engrams impaired the retrieval of contexture fear memory through TLR2/4-dependent microglia activation and spine elimination.

The expression of autophagy-related gene 7 (ATG7) in dorsal DG engrams is increased in aged mouse, leading to the activation of the surrounding microglia and the spine elimination of the engrams in a TLR2/4-dependent manner. Rac1, a mediator of forgetting, is upregulated in aged engrams and its activation promotes ATG7 expression and microglia activation to interfere with the retrieval of contexture fear memory. 
Figure
(Wiokich was not certified by peer review) is the author/funder, who has granted bioRxiv a license to display the preprint in perpetuity. It is A made available under aCC-BY 4.0 International license.
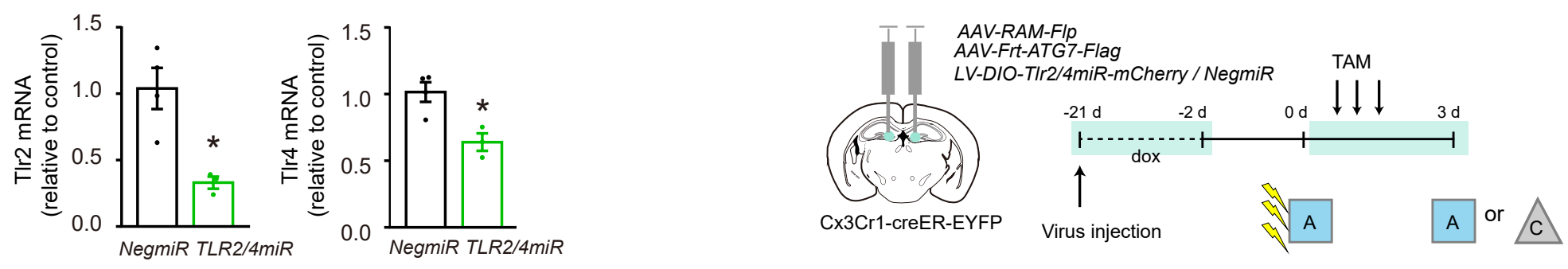

C
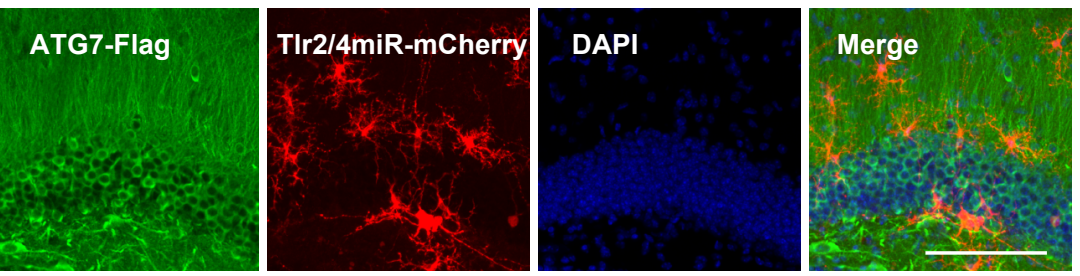

D

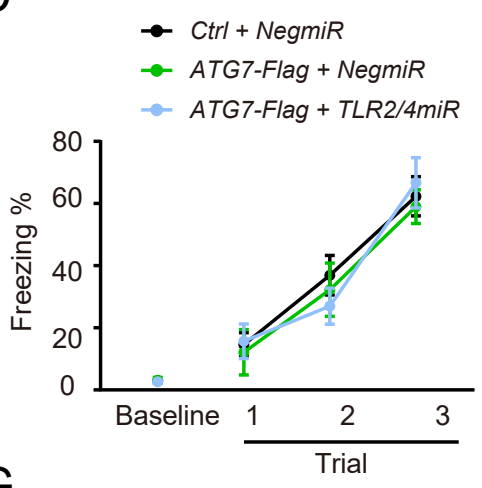

Ctrl + NegmiR

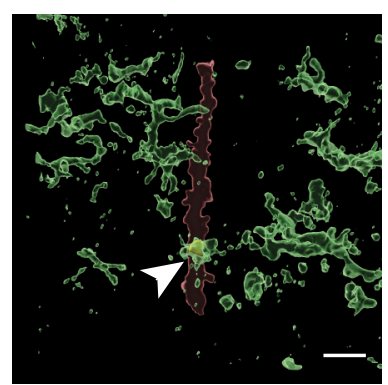

E
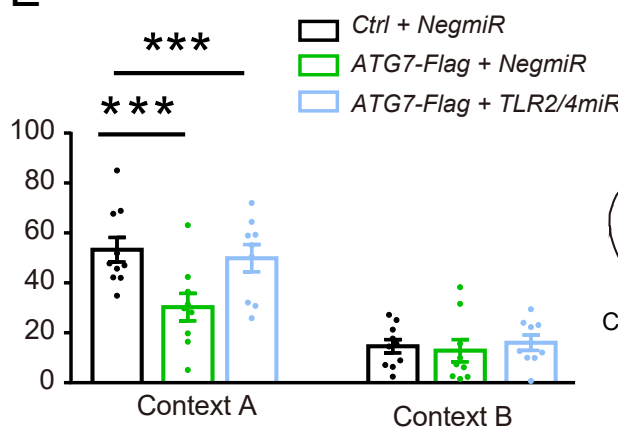

ATG7-Flag + NegmiR

ATG7-Flag + TLR2/4miR
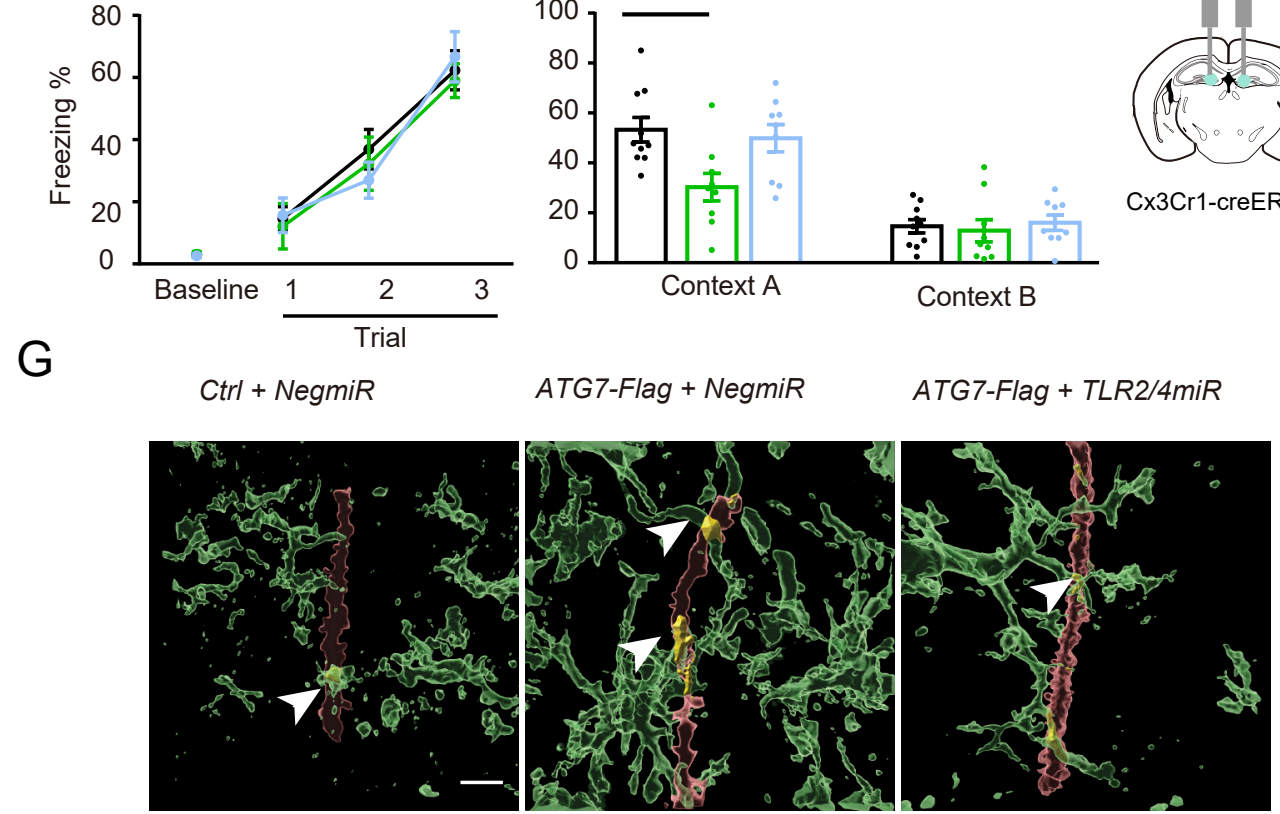

AAV-Ram-FIp

F AAV-Frt-ATG7-Flag

AAV-RAM- myrmScarlet-I-P2A-post-eGRASP

LV-DIO-TLR2/4miR-EGFP / NegmiR

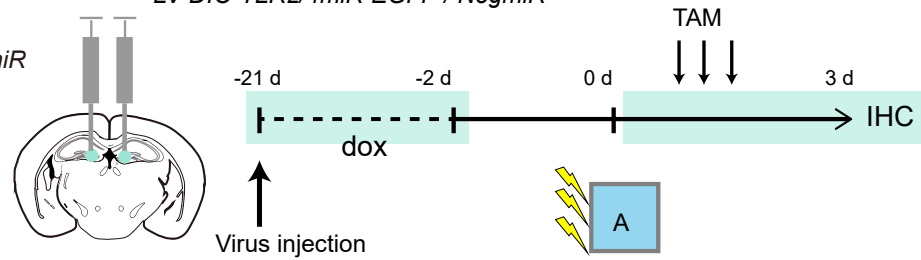

CX3Cr1-creER-EYFP
Ctrl + NegmiR

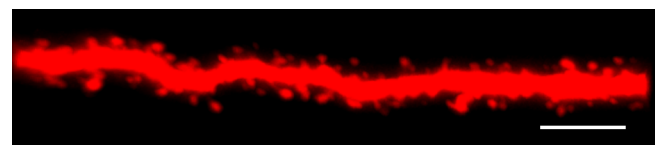

ATG7-Flag + NegmiR

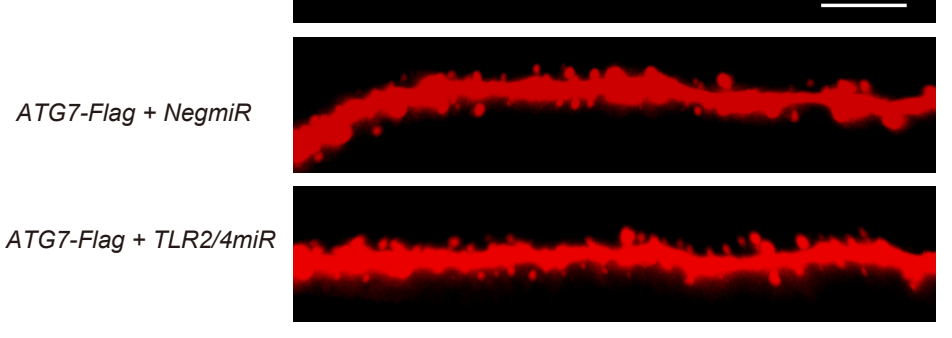

ATG7-Flag + TLR2/4miR

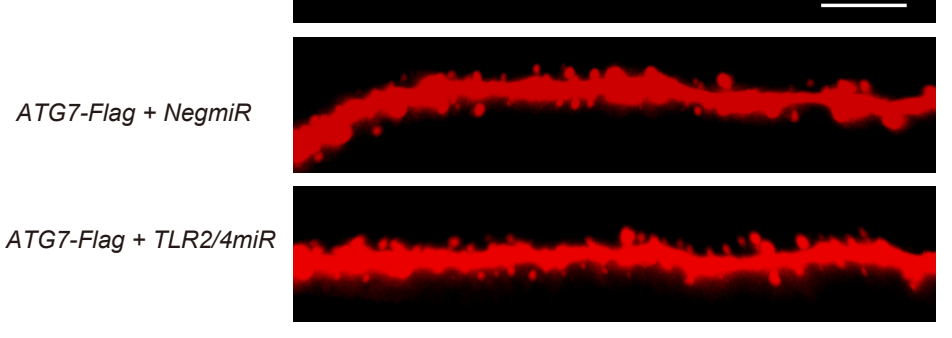

$\mathrm{H}$
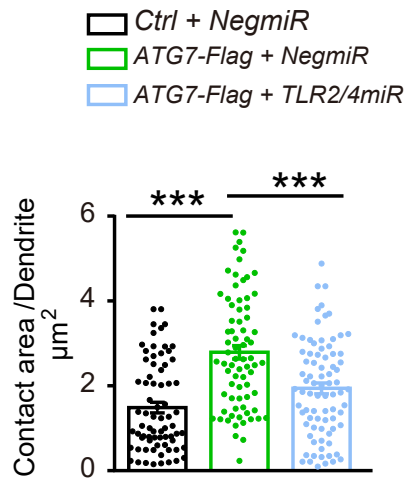

J

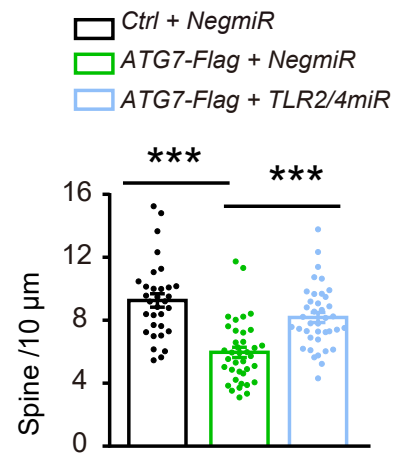




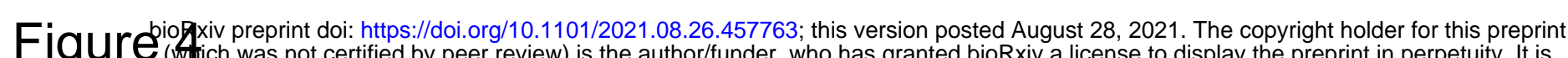
GUre (witich was not certified by peer review) is the author/funder, who has granted bioRxiv a license to display the preprint in perpetuity. It is

A

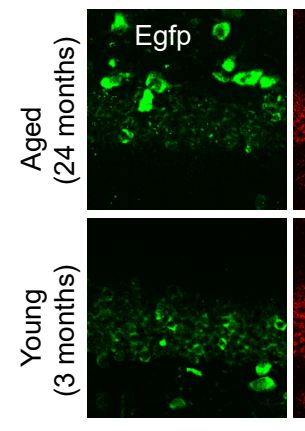

made available under aç C-BY 4.0 International license.

Rac1
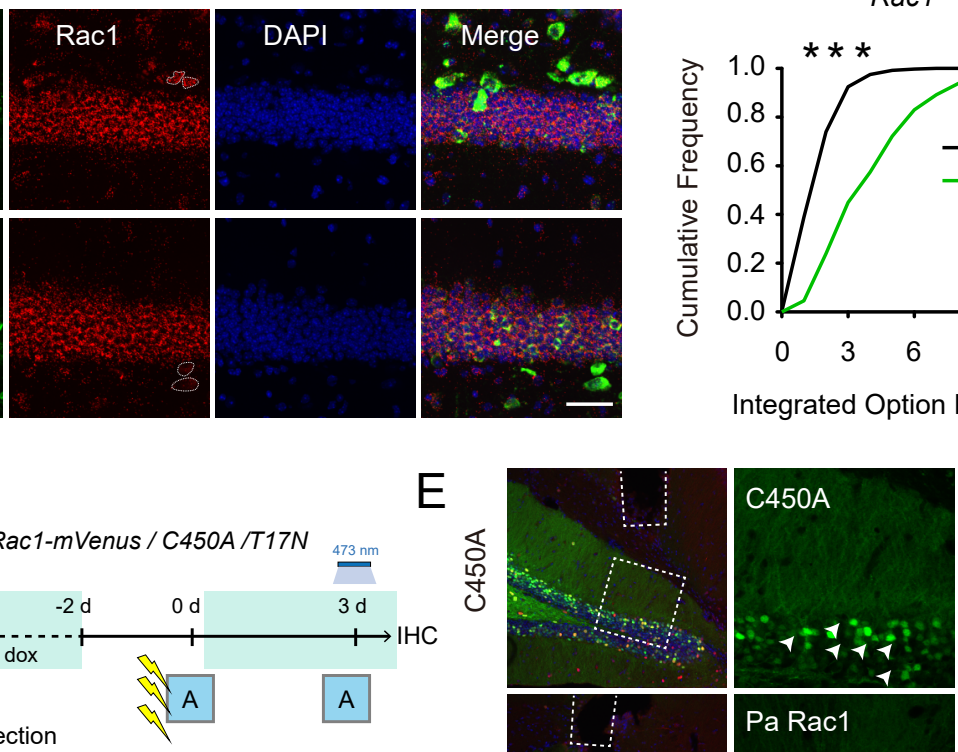

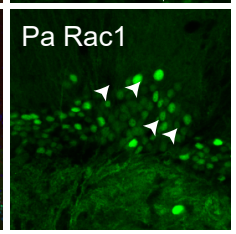

।

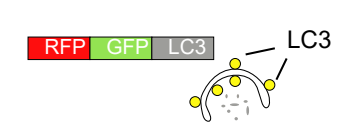

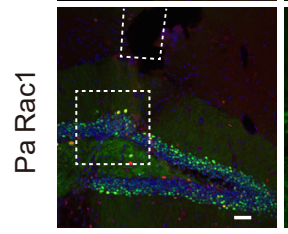

C57/BL6J

G

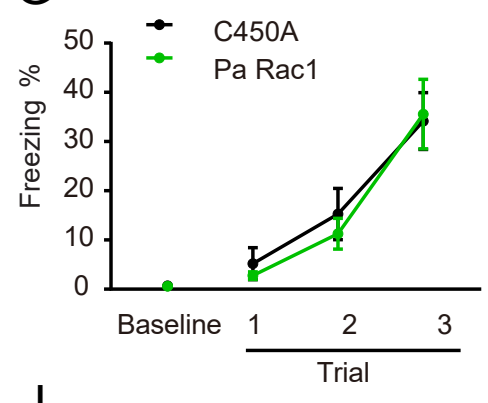

$\mathrm{J}$

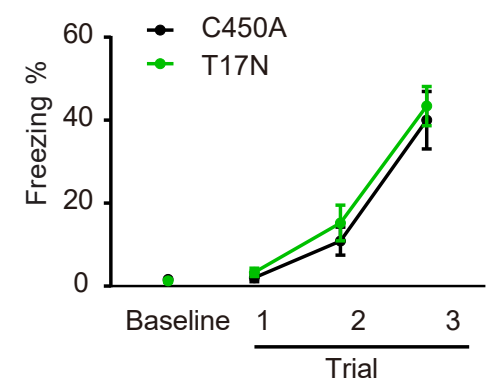

M

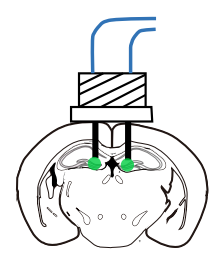

AAV-RAM-Cre

AAV-DIO-Pa-Rac1-HA

AAV-DIO-RFP-GFP-LC3

C57/BL6J Virus injection
$\mathrm{H}$

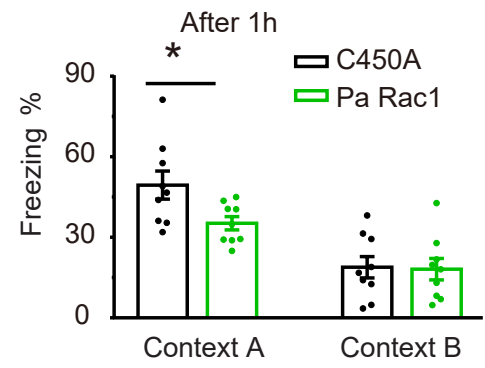

K

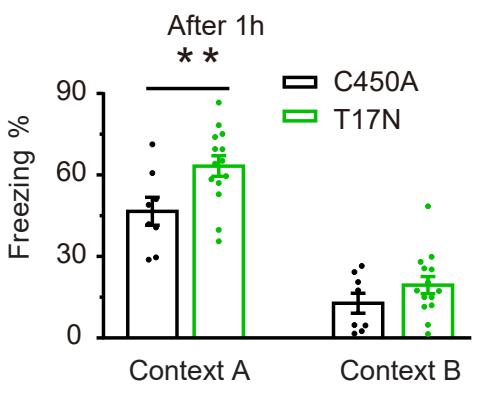

$\mathrm{N}$

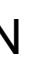

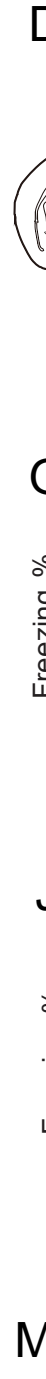

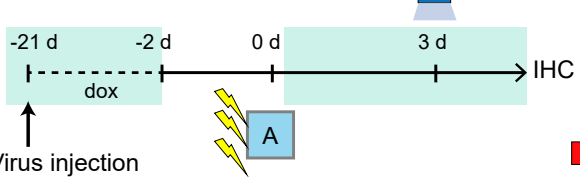

Autophagolysosome
C

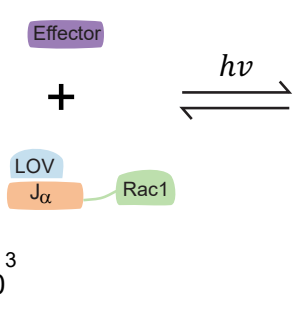

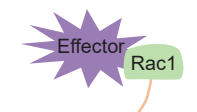

$\alpha$

$\mathrm{O}_{2}$

F

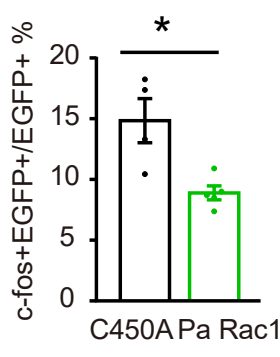

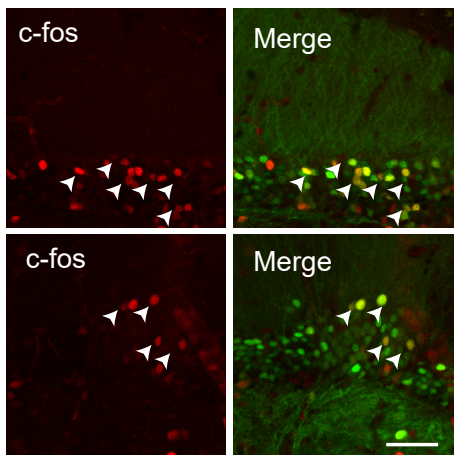

After 24h

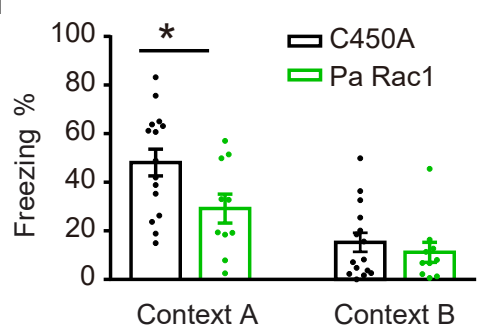

$L$

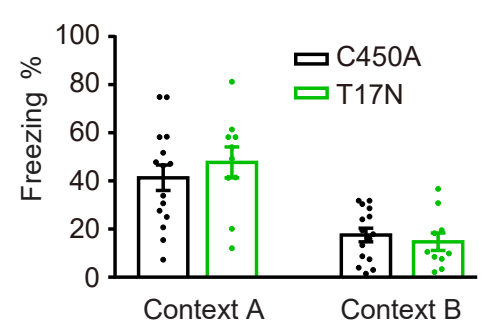

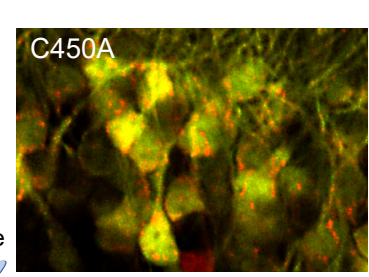

$\mathrm{O}$

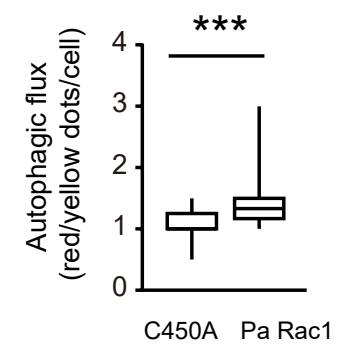




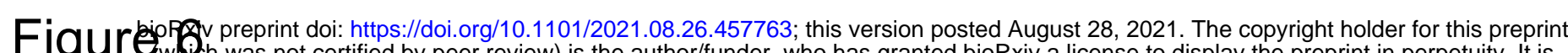

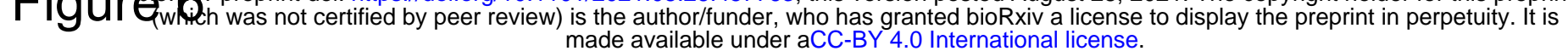
A made avaliable under $\mathrm{aCC}-\mathrm{BY} 4.0 \mathrm{O}$ international license.

A

AAV-RAM-Cre

AAV-DIO-Pa-Rac1/C450A

AAV-DIO-Atg7-shRNA-EGFP / Scramble

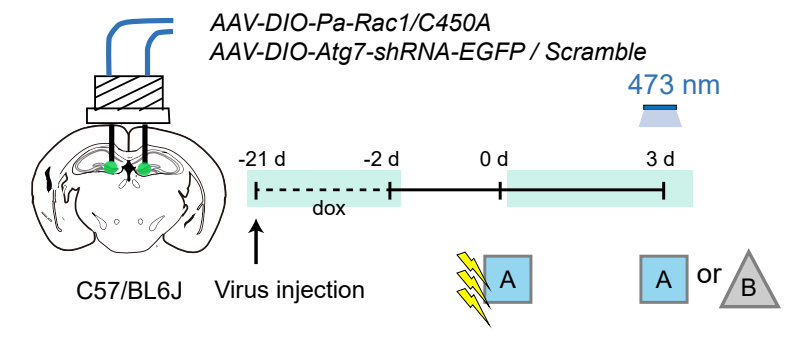

D

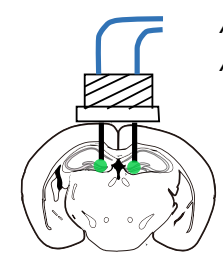

AAV-RAM-Cre

AAV-DIO-Pa-Rac1/C450A

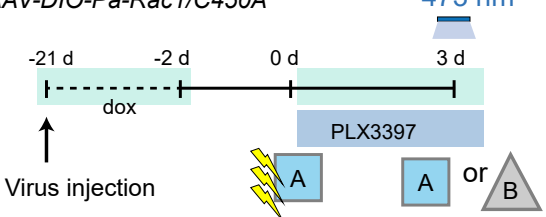

C57/BL6J
B

- C450A + Scramble

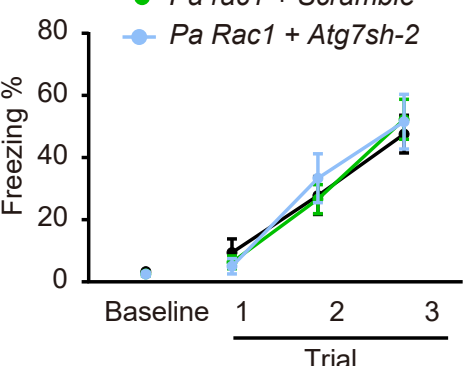

E

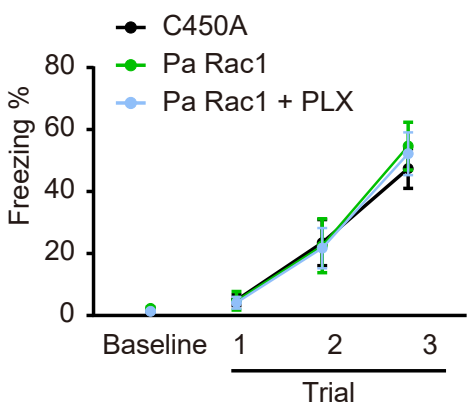

C

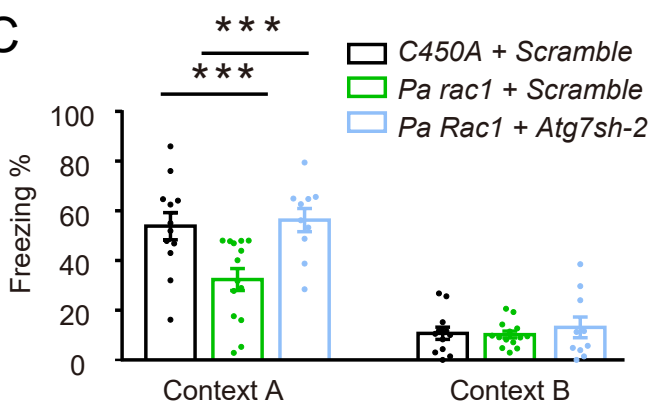

$\mathrm{F}$

$\mathrm{H}$

Elevated Plus Maze $\square$ C450A

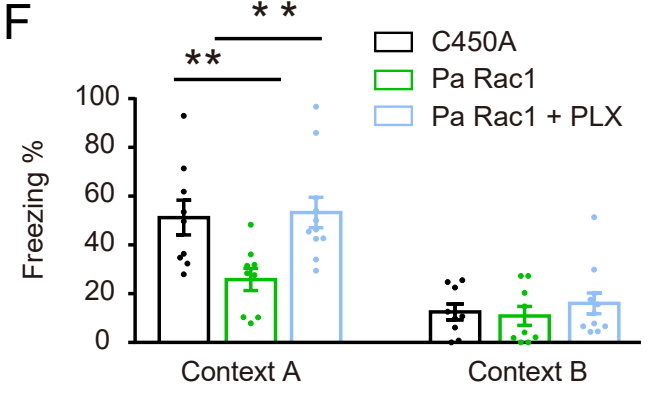

$\square$ Pa Rac1

Pa Rac1 + PLX

G Open Field
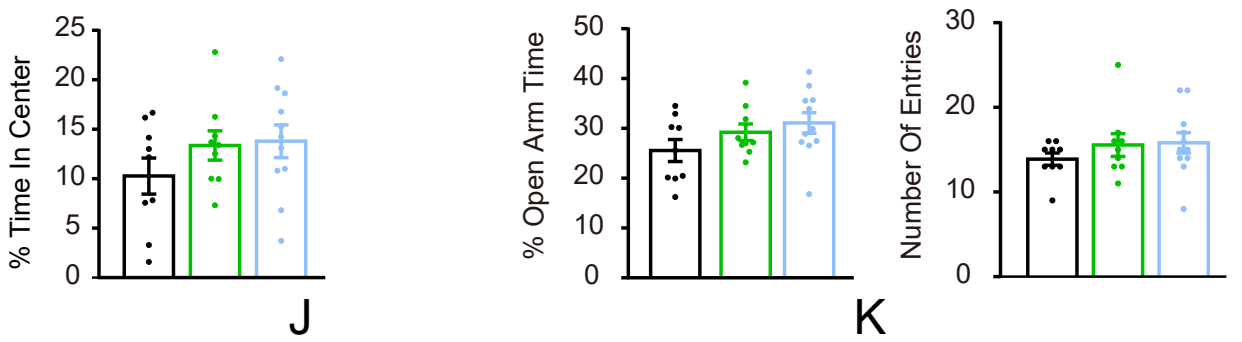

I

AAV-RAM-FIp

AAV-Frt-Pa-Rac1-HA

LV-DIO-TLR2/4miR-mCherry NegmiR

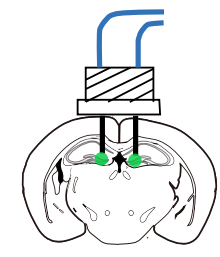

Cx3Cr1-CreER

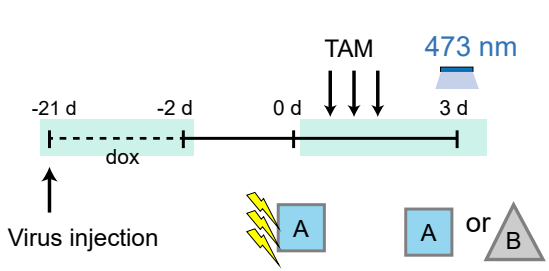

Open Field

$\mathrm{L}$
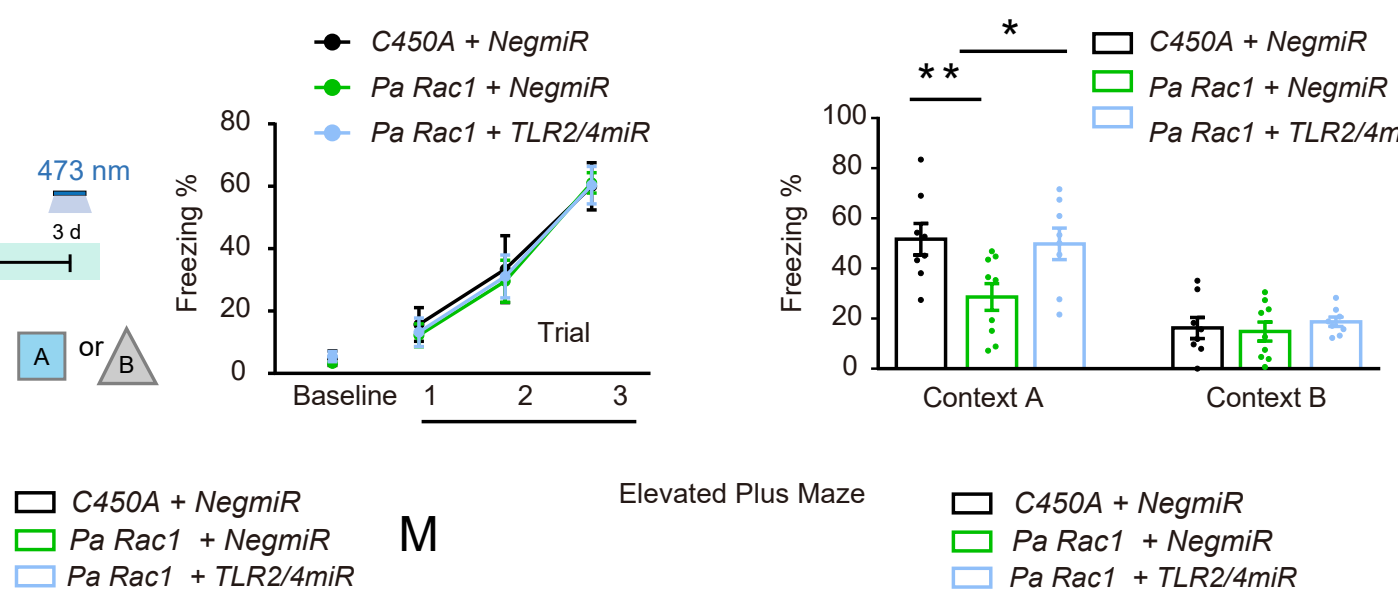

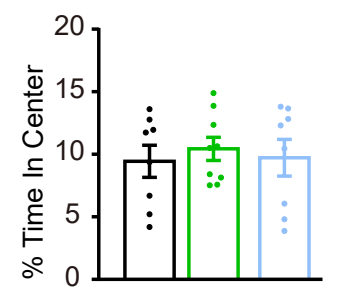

Elevated Plus Maze

$C 450 A+$ NegmiR

Pa Rac1 + NegmiR Pa Rac1 + TLR2/4miR
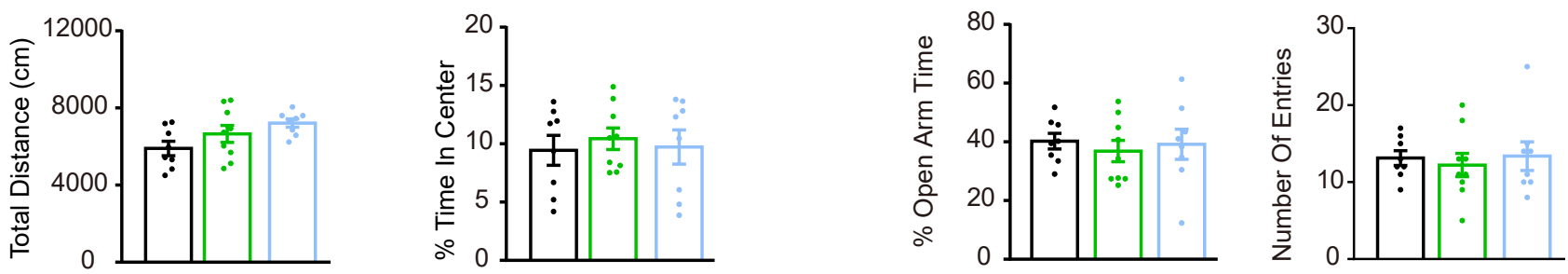
DG engram

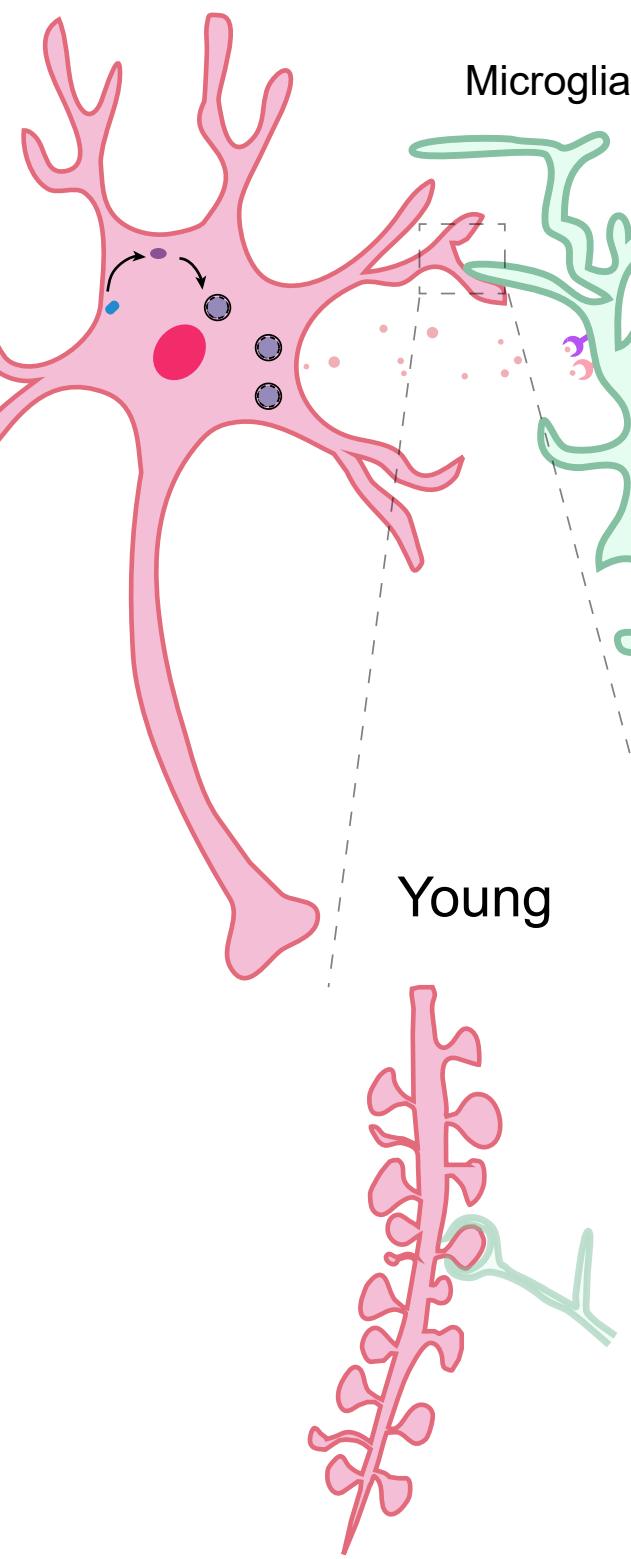

DG engram

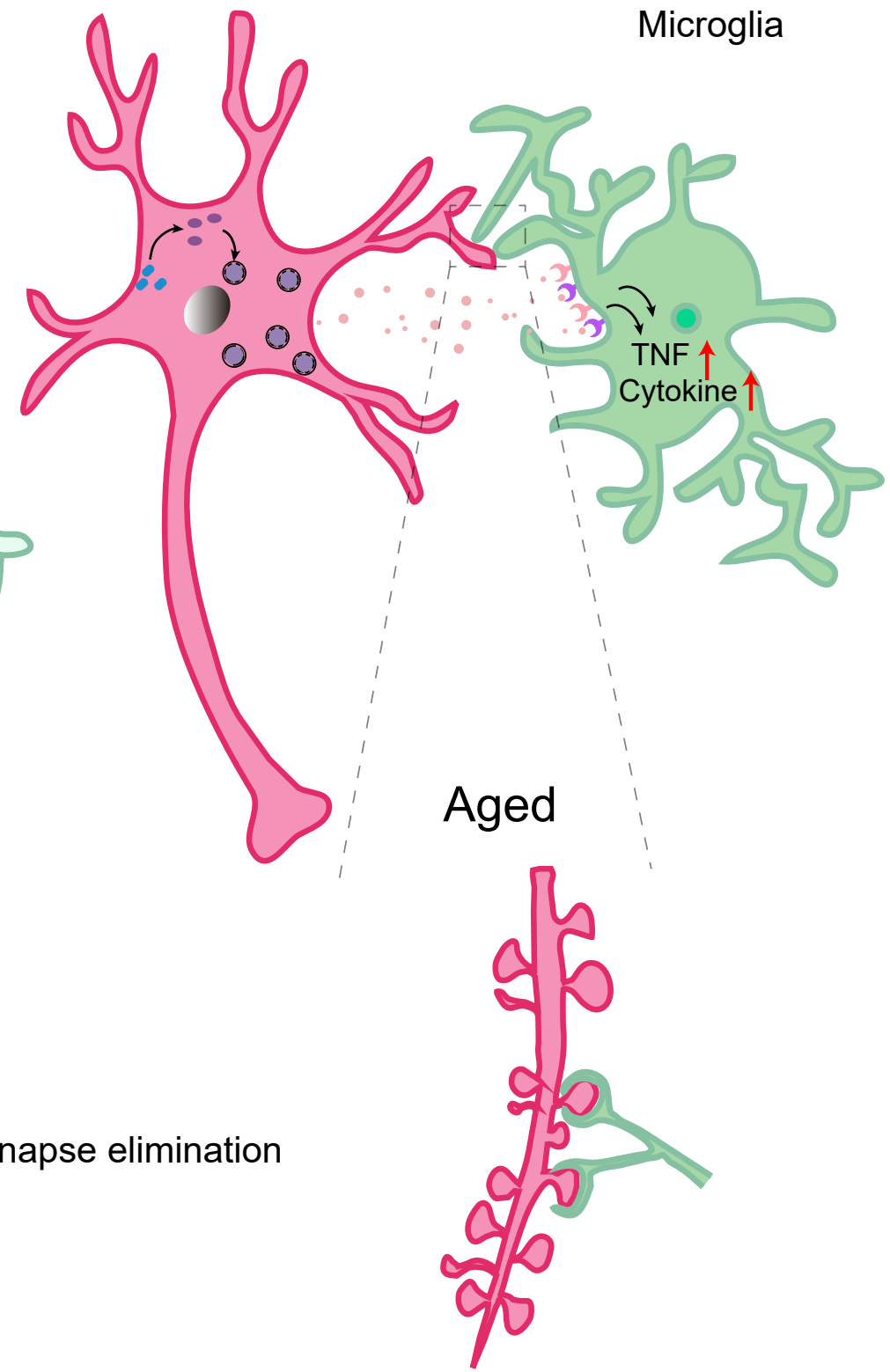

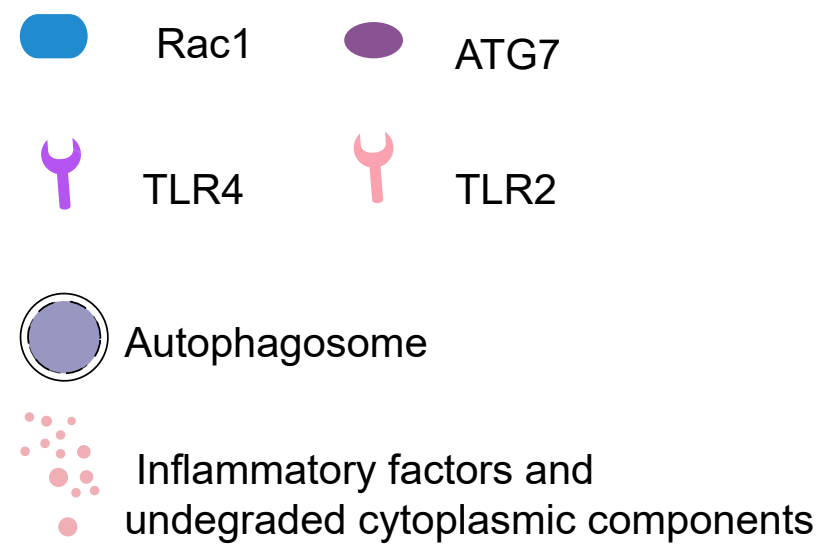

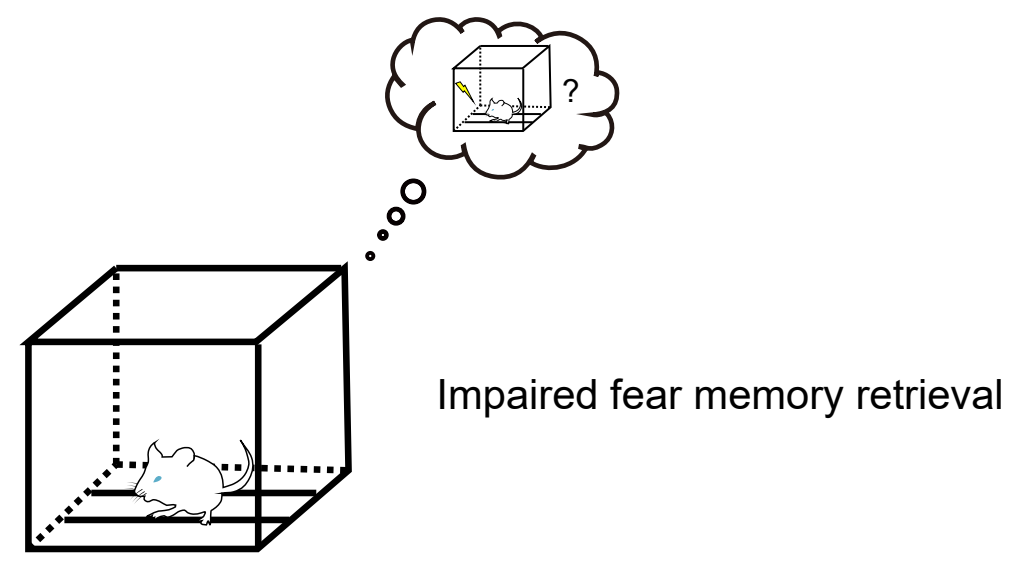

